\title{
Finite-Time Tracking Control for Nonstrict-Feedback State-Delayed Nonlinear Systems with Full-State Constraints and Unmodeled Dynamics
}

\author{
Yangang Yao $\mathbb{D},{ }^{1}$ Jieqing Tan $\mathbb{D}^{1},{ }^{1}$ and Jian $\mathrm{Wu}^{2}$ \\ ${ }^{1}$ School of Mathematics, Hefei University of Technology, Hefei 230601, China \\ ${ }^{2}$ School of Computer and Information, Anqing Normal University, Anqing 246011, China \\ Correspondence should be addressed to Jieqing Tan; jieqingtan@hfut.edu.cn
}

Received 6 September 2020; Revised 27 September 2020; Accepted 15 October 2020; Published 9 November 2020

Academic Editor: Dimitri Volchenkov

Copyright (c) 2020 Yangang Yao et al. This is an open access article distributed under the Creative Commons Attribution License, which permits unrestricted use, distribution, and reproduction in any medium, provided the original work is properly cited.

\begin{abstract}
The problem of finite-time tracking control is discussed for a class of uncertain nonstrict-feedback time-varying state delay nonlinear systems with full-state constraints and unmodeled dynamics. Different from traditional finite-control methods, a $C^{1}$ smooth finite-time adaptive control framework is introduced by employing a smooth switch between the fractional and cubic form state feedback, so that the desired fast finite-time control performance can be guaranteed. By constructing appropriate Lyapunov-Krasovskii functionals, the uncertain terms produced by time-varying state delays are compensated for and unmodeled dynamics is coped with by introducing a dynamical signal. In order to avoid the inherent problem of "complexity of explosion" in the backstepping-design process, the DSC technology with a novel nonlinear filter is introduced to simplify the structure of the controller. Furthermore, the results show that all the internal error signals are driven to converge into small regions in a finite time, and the full-state constraints are not violated. Simulation results verify the effectiveness of the proposed method.
\end{abstract}

\section{Introduction}

During the past few decades, great achievements have been proposed for uncertain nonlinear systems based on adaptive control technique, especially for pure-feedback systems (e.g., see [1-5]) and strict-feedback systems (e.g., see [6-9]) with the lower-triangular structure. Lately, the authors in [10] introduced a more general nonlinear system named nonstrict-feedback nonlinear systems. By employing the variable separation method, the tracking control problem has been well solved. Since then, many control techniques for nonstrict-feedback systems and extensions to other fields were achieved (e.g., see [11-17]).

It is known to all that many practical systems encounter the effect of the constraints, such as the temperature of chemical reactor and physical stoppages. Thus, the research about the systems with state constraints is very meaningful and necessary on account of the existence of state constraints which may undermine the stability of the system. In order to tackle the problem of state constraints, some effective control techniques (e.g., model predictive control (MPC) $[18,19]$, reference governors (RGs) [20], one-to-one nonlinear mapping (NM) [21-23], and barrier Lyapunov functions (BLFs) [24-28]) have been presented. Due to the fact that MPC and RGs require strong online computing capability to guarantee constraints, this requirement restricts their applications in engineering design. Therefore, one-to-one NM and the BLFs-based methods become the main methods to deal with the constrained nonlinear systems. There exist many significant results which focus on lower-triangular structure nonlinear systems with different constraints (e.g., input constraints [3], output constraints [24], partial-state constraints [25], and full-state constraints $[21-23,26,27])$. In addition, the rate of convergence is also an essential consideration for most practical systems. The works mentioned above only obtain asymptotic or exponential stability with infinite time, which cannot meet the requirement of finite-time control in most practical control 
systems. As a consequence, a considerable number of meaningful researches (e.g., see [28-33]) have been proposed on finite-time control for nonlinear systems. However, most of the works are to present $C^{0}$ finite-time controller by using a backstepping technique together with a nonsmooth fractional feedback design method. In order to achieve a faster convergence rate, the authors in [34] originally proposed a $C^{1}$ smooth finite-time adaptive $\mathrm{NN}$ controller by using a smooth switch between the fractional and cubic form state feedback. Moreover, there are other significant results presented in [35-41], such that two globally stable adaptive controllers were proposed in $[35,36]$. To obtain the tracking accuracy, a practical adaptive fuzzy tracking controller for a class of perturbed nonlinear systems with backlash nonlinearity has been designed in [37]. An adaptive fuzzy output-feedback tracking control technique for switched stochastic pure-feedback nonlinear systems has been presented in [38]. The authors in [39] proposed an observed-based adaptive finite-time tracking control technique for a class of nonstrict-feedback nonlinear systems with input saturation. An adaptive finite-time output-feedback controller for switched pure-feedback nonlinear systems with average dwell time has been given in [40]. A decentralized event-triggered controller for interconnected systems with unknown disturbances has been proposed in [41].

Furthermore, due to the fact that unmodeled dynamics can severely degrade the closed-loop system performance, dealing with the effects of unmodeled dynamics is essential for practical nonlinear control systems. Therefore, several results were proposed by employing backstepping or DSC in $[4,21-23,42-47]$. Generally, unmodeled dynamics was disposed by introducing a dynamic signal in $[4,21-23,42-46]$ or a Lyapunov function description in [47].

In addition, time delays frequently occur in some practical engineering systems. As stated in [48], their existence can deteriorate the transient performance and even can destroy the stability of the control systems. Thus, the research on nonlinear time-delay systems has become one of the hot topics and some meaningful results have been achieved during the past decades [49-53]. For uncertain nonlinear time-delay systems, the effective controller was developed originally in [50] by combining the backstepping technique with Lyapunov-Krasovskii functionals. Soon afterward, this method was extended to nonlinear strictfeedback time-delay system with unknown control gain functions [51] and uncertain multi-input/multi-output nonlinear systems with time delays [52]. Later, some improved control schemes based on [50] were proposed (e.g., see $[35,53,54])$.

Although many significant research results on adaptive neural network control for uncertain nonstrict-feedback systems have been obtained in [11-17], their considered systems did not include unmodeled dynamics or full-state constraints. In [21-28], the effective controllers have been designed for the lower-triangular structure nonlinear systems with state constraints and unmodeled dynamics, but their considered systems did not include state delay and their control methods may be invalid to nonstrict-feedback systems on account of subsystem function which contains the whole state variables. Furthermore, the above-mentioned control methods only obtain asymptotic or exponential stability with infinite time. To the best knowledge of the authors, finite-time tracking control for a class of uncertain nonstrict-feedback time-varying state-delayed nonlinear systems with full-state constraints and unmodeled dynamics has not been fully discussed in the literature, which is still open and remains unsolved. In this paper, we are committed to solving the problem mentioned above. The main contributions of the paper are summarized as follows:

(i) In contrast to the existing results reported in $[21-28,47]$ where the control methods have been proposed for nonlinear strict-feedback or purefeedback systems with state or output constraints and unmodeled dynamics, a generalization of the results is proposed for a class of nonstrict-feedback state delay systems with state constraints and unmodeled dynamics of which the subsystem function contains the whole state variables. To the best of authors' knowledge, it is the first time to develop an adaptive DSC method for uncertain nonstrict-feedback state delay systems with state constraints and unmodeled dynamics.

(ii) Different from the finite-control methods in [31-33], a $C^{1}$ smooth finite-time adaptive control framework is introduced by employing a smooth switch between the fractional and cubic form state feedback reported in [34], so that the desired fast finite-time control performance can be guaranteed. Moreover, unmodeled dynamics is coped with by introducing a dynamical signal and the uncertain terms produced by time-varying state delays are compensated for by constructing appropriate Lyapunov-Krasovskii functionals. The results show that all the error signals are driven to converge into small regions in a finite time, and the full-state constraints are never violated.

The remainder of this paper is organized as follows. In Section 2, the problem formulation and preliminaries are presented. Adaptive DSC design and stability analysis are given in Section 3. Simulation results verify the effectiveness of the proposed control approach in Section 4, followed by Section 5, which concludes this paper.

Notation. In this paper, $R$ denotes a set of real numbers, $R^{+}$ denotes a set of nonnegative real numbers, $R^{m \times n}$ denotes a set of $m \times n$ real matrices, $R^{n}$ denotes a set of $\mathrm{n}$-dimensional real vectors, $\sup (\cdot)$ denotes the least upper bound, $\|\cdot\|$ denotes 2-norm of a vector or matrix, $|\cdot|$ denotes an absolute value of a real number $\cdot, \exp (\cdot)$ denotes an exponential function of $\cdot$, and $\log (\cdot)$ denotes the natural logarithm of $\cdot$

\section{Problem Formulation and Preliminaries}

2.1. Problem Statement. Consider a class of uncertain nonstrict-feedback state-delayed nonlinear systems with unmodeled dynamics for $i=1,2, \ldots, n-1$ in the following form: 


$$
\left\{\begin{array}{l}
\dot{\xi}=q(\xi, x, t), \\
\dot{x}_{i}=f_{i}(x)+g_{i}\left(\bar{x}_{i}\right) x_{i+1}+\delta_{i}(\xi, x, t)+d_{i}\left(\bar{x}_{i}\left(t-T_{i}(t)\right)\right), \\
\dot{x}_{n}=f_{n}(x)+g_{n}\left(\bar{x}_{n}\right) u+\delta_{n}(\xi, x, t)+d_{n}\left(\bar{x}_{n}\left(t-T_{n}(t)\right)\right), \\
y=x_{1},
\end{array}\right.
$$

where $x=\left[x_{1}, x_{2}, \ldots, x_{n}\right]^{T} \in R^{n}$ is the state vector, $\xi \in R^{n_{0}}$ is the unmodeled dynamics, and $u, y, T_{i}(t)$ denote the system input, the system output, and the unknown timevarying delays, respectively. $f_{i}(x), g_{i}\left(\bar{x}_{i}\right)$, and $d_{i}\left(\bar{x}_{i}\left(t-T_{i}(t)\right)\right)$ are the unknown smooth functions. Let $\bar{x}_{i}=\left[x_{1}, x_{2}, \ldots, x_{i}\right]^{T}$ and $\delta_{i}(\xi, x, t)$ be the unknown uncertain disturbances. All the states $x_{i}$ are required to remain in the sets $\Omega_{x_{i}}=\left\{x_{i}:\left|x_{i}\right|<k_{c_{i}}\right\}$, where $k_{c_{i}}$ are positive constants.

Remark 1. System (1) is called a nonstrict-feedback form in which the system function $f_{i}(\cdot)$ and its bounding function contain all the state variables [10]. Apparently, strict-feedback and pure-feedback structures are the special cases of system (1). The methods proposed in [21-28, 31-33, 47] cannot be directly applied to system (1) on account of its nonstrict-feedback structure.

The control objective of this paper is to construct an adaptive NN controller $u(t)$ to make sure that the output $y$ follows the desired trajectory $y_{r}$ in a finite time, while every state $x_{i} \in \Omega_{x_{i}}$ is never violated.

2.2. RBFNN Approximation. In this paper, for $i=1, \ldots, n$, the unknown smooth nonlinear functions $\bar{F}_{i}\left(Z_{i}\right): R^{m} \longrightarrow R$ will be approximated on a compact set $\Omega_{i} \subset R^{m}$ by the following RBFNN:

$$
\bar{F}_{i}\left(Z_{i}\right)=W_{i}^{T} S_{i}\left(Z_{i}\right)+\varepsilon_{i}\left(Z_{i}\right)
$$

where $Z_{i}, W_{i}, l$ denote input vectors, weight vectors, and NN node number, respectively. $\varepsilon_{i}\left(Z_{i}\right)$ are the $\mathrm{NN}$ inherent approximation errors which are bounded over the compact sets; that is, $\varepsilon_{i}\left(Z_{i}\right) \leq \varepsilon_{i}$, where $\epsilon_{i}$ are unknown constants and $S_{i}\left(Z_{i}\right)=\left[s_{1}\left(Z_{i}\right), \ldots, s_{l}\left(Z_{i}\right)\right]^{T}: \Omega_{i} \longrightarrow R^{l}$ are known smooth vector functions with $s_{q}\left(Z_{i}\right)$ being chosen as the commonly used Gaussian functions, which have the form

$$
s_{q}\left(Z_{i}\right)=\exp \left[\frac{-\left(Z_{i}-\mu_{q}\right)^{T}\left(Z_{i}-\mu_{q}\right)}{\eta_{q}^{2}}\right], \quad q=1, \ldots, l,
$$

where $\mu_{q}=\left[\mu_{q 1}, \ldots, \mu_{q m}\right]^{T}$ is the center vector and $\eta_{q}$ is the spreads of the Gaussian function. The optimal weight vector $W_{i}$ is defined as

$$
W_{i}=\underset{\widehat{W}_{i} \in R^{l}}{\arg \min }\left\{\sup _{Z_{i} \in \Omega_{i}}\left|F\left(Z_{i}\right)-\widehat{W}_{i}^{T} S(Z)\right|\right\},
$$

where $\widehat{W}_{i}$ is the estimate of $W_{i}$.

\subsection{Key Definition and Lemmas}

Definition 1 (see [21]). The unmodeled dynamics $\xi$ is said to be exponentially input-state-practically stable (exp-ISpS), that is, for system $\dot{\xi}=q(\xi, x, t)$, if there exist functions $\bar{\alpha}_{1}, \bar{\alpha}_{2}$ of class $K_{\infty}$ and a Lyapunov function $V(\xi)$, such that

$$
\bar{\alpha}_{1}(\|\xi\|) \leq V(\xi) \leq \bar{\alpha}_{2}(\|\xi\|)
$$

and there exist two constants $c>0, d \geq 0$ and a class $K_{\infty}$ function $\gamma$, such that

$$
\frac{\partial V(\xi)}{\partial \xi} q(\xi, x, t) \leq-c V(\xi)+\gamma\left(\left|x_{1}\right|\right)+d, \quad \forall t \geq 0
$$

where $c$ and $d$ are known positive constants and $\gamma(\cdot)$ is a known function of class $K_{\infty}$.

Lemma 1 (see [21]). If $V$ is an exp-ISpS Lyapunov function for a system $\dot{\xi}=q(\xi, x, t)$, that is, (5) and (6) hold, then, for any constant $\bar{c} \in(0, c)$, any initial instant $t_{0}>0$, any initial condition $\xi_{0}=\xi\left(t_{0}\right), r_{0}>0$, and any continuous function $\bar{\gamma}$, such that $\bar{\gamma}\left(\left|x_{1}\right|\right) \geq \gamma\left(\left|x_{1}\right|\right)$, there exist a finite $T_{0}=\max \left\{0, \log \left[\left(V\left(\xi_{0}\right) / r_{0}\right) /(c-\bar{c})\right]\right\} \geq 0, \quad$ a nonnegative function $D\left(t_{0}, t\right)$ defined for all $t \geq t_{0}$, and a signal described by

$$
\dot{r}=-\bar{c} r+\bar{\gamma}\left(\left\|x_{1}\right\|\right)+d, r\left(t_{0}\right)=r_{0},
$$

such that $D\left(t_{0}, t\right)=0$ for $t \geq t_{0}+T_{0}$ and $V(\xi) \leq r(t)+D\left(t_{0}, t\right) \quad$ with $\left.D\left(t_{0}, t\right)=\max \left\{0, e^{-c\left(t-t_{0}\right)} V\left(z_{0}\right)-e^{-\bar{c}\left(t-t_{0}\right)} r_{0}\right)\right\}$.

Lemma 2 (see [11]). Let $S(Z)$ be the basis function vector of an RBFNN and $Z$ be the input vector, where $S(Z)=\left[s_{1}(Z), \ldots, s_{l}(Z)\right]^{T}$ and $Z=\left[z_{1}, \ldots z_{n}\right]^{T}$. For any positive integer $m \leq n$, let $Z_{m}=\left[z_{1}, \ldots, z_{m}\right]^{T}$, and the following inequality holds:

$$
\|S(Z)\|^{2} \leq\left\|S\left(Z_{m}\right)\right\|^{2} .
$$

Lemma 3 (see [55]). For any real numbers $\zeta_{1}>0, \zeta_{2}>0$ and $0<h<1$, an extended Lyapunov condition of finite-time stability can be given in the form of fast terminal sliding mode as $\dot{V}(x)+\zeta_{1} V(x)+\zeta_{2} V^{h}(x) \leq 0$; then, $V(x)$ is in fast finitetime convergent with a finite settling time $T^{*} \leq\left(1 / \zeta_{1}(1-\right.$ h) $) \log \left(\left(\zeta_{1} V^{1-h}\left(x_{0}\right)+\zeta_{2}\right) / \zeta_{2}\right)$.

Lemma 4 (see [56]). For $x, y \in R$, if $0<h=h_{2} / h_{1}<1$, where $h_{1}, h_{2}>0$ are odd integers, then $x y^{h} \leq-\varsigma_{1} x^{1+h}+\varsigma_{2}(x+$ $y)^{1+h}$, where $\varsigma_{1}=(1 /(1+h))\left(2^{h-1}-2^{(h-1)(h+1)}\right)$ and $\varsigma_{2}=$ $(1 /(1+h))\left(1+(2 h /(1+h))+\left(2^{-(h-1)^{2}(h+1)} /(1+h)\right) 2^{h-1}\right)$. 
Lemma 5 (see [34]). Consider the dynamic system

$$
\dot{\phi}(t)=-l_{1} \phi(t)-l_{2} \phi^{h}(t)+\varrho(t),
$$

where $\phi(t) \in R, 0<h=\left(h_{2} / h_{1}\right)<1 \quad\left(h_{1}\right.$ and $h_{2}$ are positive odd integers), $l_{1}$ and $l_{2}$ are positive constants, and $\varrho(t)$ is a positive function. Then, for any given bounded initial condition $\phi(0) \geq 0$, one has that $\phi(t) \geq 0, \forall t \geq 0$.

Lemma 6 (see [57]). For $x_{i} \in R, i=1,2, \ldots, n$, and $0<h \leq 1$, then $\left(\sum_{i=1}^{n}\left|x_{i}\right|\right)^{h} \leq \sum_{i=1}^{n}\left|x_{i}\right|^{h} \leq n^{1-h}\left(\sum_{i=1}^{n}\left|x_{i}\right|\right)^{h}$.

To obtain the control objective, the following assumptions are needed.

Assumption 1. The unmodeled dynamics $\xi$ is exp-ISpS.

Assumption 2. There exist unknown nonnegative continuous functions $\varphi_{i 1}$ and nondecreasing continuous functions $\varphi_{i 2}$ such that

$$
\left|\delta_{i}(\xi, x, t)\right| \leq \varphi_{i 1}\left(\left\|\bar{x}_{i}\right\|\right)+\varphi_{i 2}(\|\xi\|), \quad \forall(\xi, x, t) \in R^{n_{0}} \times R^{n} \times R^{+},
$$

where $\varphi_{i 2}(0)=0, \quad i=1, \ldots, n$.

Remark 2. From Definition 1 and Assumption 1, we have $\|\xi\| \leq \bar{\alpha}_{1}^{-1}(V(\xi))$. According to Lemma 1, there exists a positive constant $D_{0}$ such that $\|\xi\| \leq \bar{\alpha}_{1}^{-1}\left(r+D_{0}\right), \forall t \geq 0$. This inequality will be used to cope with the uncertain terms in the following controller design.

Assumption 3. The sign of $g_{i}\left(\bar{x}_{i}\right)$ is known, and there exist some unknown positive constants $a_{i}$ and $b_{i}$ such that $0<b_{i} \leq\left|g_{i}\left(\bar{x}_{i}\right)\right| \leq a_{i}$. Without loss of generality, this paper assumes that $g_{i}\left(\bar{x}_{i}\right)>0$.

Assumption 4. The reference trajectory $y_{r}(t)$ and its derivatives about time $\dot{y}_{r}$ and $\ddot{y}_{r}$ are in a bounded region $\Omega_{d}$, and there exists a known constant $A_{0}$, such that $\left|y_{r}\right| \leq A_{0}<k_{c 1}$.

Assumption 5. The unknown continuous functions $d_{i}\left(\bar{x}_{i}\left(t-T_{i}(t)\right)\right)$ satisfy the following inequality:

$$
d_{i}\left(\bar{x}_{i}\left(t-T_{i}(t)\right)\right) \leq \sum_{j=1}^{i} \rho_{i j}\left(x_{j}\left(t-T_{j}(t)\right)\right),
$$

and the time-varying state delays $T_{i}(t)$ satisfy the inequalities $0 \leq T_{i}(t) \leq T_{\max }$ and $\dot{T}_{i}(t) \leq \bar{T}_{\max }<1$, where $\rho_{i j}\left(x_{j}\left(t-T_{j}(t)\right)\right)$ are unknown positive smooth functions and $T_{\max }$ and $\bar{T}_{\max }$ are unknown constants.

\section{Adaptive DSC Design and Stability Analysis}

3.1. Adaptive DSC Design. Similar to traditional backstepping, the backstepping-design procedure with $n$ steps is developed to construct the adaptive neural controller in this part.

By using the backstepping technique, the proposed adaptive DSC scheme contains $n$ steps as follows.

Step 1. Define the first surface error $z_{1}=x_{1}-y_{r}$; the time derivative of $z_{1}$ is defined as

$$
\dot{z}_{1}=f_{1}(x)+g_{1}\left(\bar{x}_{1}\right) x_{2}+\delta_{1}(\xi, x, t)+d_{1}\left(\bar{x}_{1}\left(t-T_{1}(t)\right)\right)-\dot{y}_{r} .
$$

The virtual control law $\alpha_{1}$ and the update law for $\dot{\widehat{\widehat{\omega}}}_{1}$ are designed as

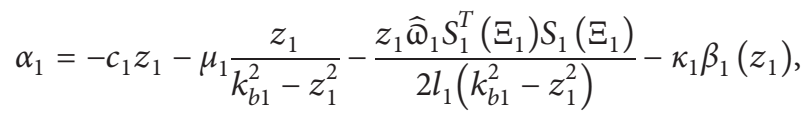

$$
\dot{\widehat{\widehat{\omega}}}_{1}=\rho_{1}\left(-\sigma_{11} \widehat{\mathrm{\omega}}_{1}-\sigma_{12} \widehat{\mathrm{\omega}}_{1}^{h}+\frac{z_{1}^{2} S_{1}^{T}\left(\Xi_{1}\right) S_{1}\left(\Xi_{1}\right)}{2 l_{1}\left(k_{b 1}^{2}-z_{1}^{2}\right)^{2}}\right),
$$

where $c_{1}, \mu_{1}, k_{b 1}, \kappa_{1}, \rho_{1}, \sigma_{11}, \sigma_{12}, l_{1}$ are positive design parameters, $\widehat{\widehat{\omega}}_{1}$ is an estimate of $\Phi_{1}, \widetilde{\omega}_{1}=\Phi_{1}-b_{1} \widehat{\widehat{\omega}}_{1}$, $\Phi_{1}=\left\|W_{1}\right\|^{2}, b_{1}$ is defined in Assumption 3. $\beta_{1}\left(z_{1}\right)$ is defined as

$$
\beta_{1}\left(z_{1}\right)= \begin{cases}z_{1}^{h}\left(k_{b 1}^{2}-z_{1}^{2}\right)^{(1-h / 2)}, & \text { if }\left|z_{1}\right| \geq \tau_{1}, \\ \iota_{11} z_{1}+\iota_{12} z_{1}^{3}, & \text { if }\left|z_{1}\right|<\tau_{1},\end{cases}
$$

where $0<h=\left(h_{1} / h_{2}\right)<1, h_{1}$ and $h_{2}$ are the positive odd integers, $\quad \iota_{11}=\tau_{1}^{h-1}\left(k_{b 1}^{2}-\tau_{1}^{2}\right)^{(1-(h / 2))}-\iota_{12} \tau_{1}^{2}, \iota_{12}=\left(1 / 2 \tau_{1}^{3}\right)$ $(h-1) \tau_{1}^{h}\left[\left(k_{b 1}^{2}-\tau_{1}^{2}\right)^{(1-(h / 2))}+\tau_{1}^{2}\left(\left(k_{b 1}^{2}-\tau_{1}^{2}\right)^{-(1+(h / 2))}\right], \quad\right.$ and $\tau_{1}<k_{b 1}$ is a small positive constant.

Consider the BLF candidate $V_{z_{1}}$ as

$$
V_{z_{1}}=\frac{1}{2} \log \frac{k_{b 1}^{2}}{k_{b 1}^{2}-z_{1}^{2}}+\frac{1}{2 b_{1} \rho_{1}} \widetilde{\Phi}_{1}^{2} .
$$

Obviously, $V_{z_{1}}$ is positive definite and continuously differentiable. Based on Assumptions 2 and 5 and Young's inequality, we obtain the time derivative of $V_{z_{1}}$ as follows: 


$$
\begin{aligned}
& \dot{V}_{z_{1}}=\frac{z_{1}}{k_{b 1}^{2}-z_{1}^{2}}\left[f_{1}(x)+g_{1}\left(\bar{x}_{1}\right) x_{2}+\delta_{1}(\xi, x, t)+d_{1}\left(\bar{x}_{1}\left(t-T_{1}(t)\right)\right)-\dot{y}_{r}\right]-\frac{1}{\rho_{1}} \widetilde{\Phi}_{1} \dot{\widehat{\Phi}}_{1}, \\
& \leq \frac{z_{1}}{k_{b 1}^{2}-z_{1}^{2}}\left[f_{1}(x)+g_{1}\left(\bar{x}_{1}\right) x_{2}-\dot{y}_{r}\right]+\frac{\left|z_{1}\right|}{k_{b 1}^{2}-z_{1}^{2}}\left[\left[\varphi_{11}\left(\left\|\bar{x}_{1}\right\|\right)+\varphi_{12}\left(\bar{\alpha}_{1}^{-1}\left(r+D_{0}\right)\right)\right]+\rho_{11}\left(x_{1}\left(t-T_{1}(t)\right)\right)\right]-\frac{1}{\rho_{1}} \widetilde{\omega}_{1} \dot{\hat{\omega}}_{1}, \\
& \leq \frac{z_{1}}{k_{b 1}^{2}-z_{1}^{2}}\left[f_{1}(x)+g_{1}\left(\bar{x}_{1}\right) x_{2}-\dot{y}_{r}\right]+\frac{z_{1}^{2}}{\left(k_{b 1}^{2}-z_{1}^{2}\right)^{2}}\left[\varphi_{11}\left(\left\|\bar{x}_{1}\right\|\right)+\varphi_{12}\left(\bar{\alpha}_{1}^{-1}\left(r+D_{0}\right)\right)\right]^{2}+\frac{1}{4}, \\
& +\frac{z_{1}^{2}}{2\left(k_{b 1}^{2}-z_{1}^{2}\right)^{2}}+\frac{1}{2} \rho_{11}^{2}\left(x_{1}\left(t-T_{1}(t)\right)\right)-\frac{1}{\rho_{1}} \widetilde{\mathrm{\omega}}_{1} \dot{\widehat{\mathrm{\omega}}}_{1}, \\
& \leq \frac{z_{1}}{k_{b 1}^{2}-z_{1}^{2}}\left[f_{1}(x)+g_{1}\left(\bar{x}_{1}\right) x_{2}+\frac{z_{1}}{k_{b 1}^{2}-z_{1}^{2}}\left[\varphi_{11}\left(\left\|\bar{x}_{1}\right\|\right)+\varphi_{12}\left(\bar{\alpha}_{1}^{-1}\left(r+D_{0}\right)\right)\right]^{2}+\frac{z_{1}}{2\left(k_{b 1}^{2}-z_{1}^{2}\right)}-\dot{y}_{r}\right] \\
& +\frac{1}{4}+\frac{1}{2} \rho_{11}^{2}\left(x_{1}\left(t-T_{1}(t)\right)\right)-\frac{1}{\rho_{1}} \widetilde{\Phi}_{1} \dot{\widehat{\widehat{\omega}}}_{1} \\
& \leq \frac{z_{1}}{k_{b 1}^{2}-z_{1}^{2}}\left[\bar{F}_{1}\left(Z_{1}\right)+g_{1}\left(\bar{x}_{1}\right) x_{2}\right]+\frac{1}{4}+\frac{1}{2} \rho_{11}^{2}\left(x_{1}\left(t-T_{1}(t)\right)\right)-\frac{1}{\rho_{1}} \widetilde{\omega}_{1} \dot{\widehat{\omega}}_{1}, \\
& =\frac{z_{1}}{k_{b 1}^{2}-z_{1}^{2}}\left[\bar{F}_{1}\left(Z_{1}\right)+g_{1}\left(\bar{x}_{1}\right)\left(z_{2}+y_{2}+\alpha_{1}\right)\right]+\frac{1}{4}+\frac{1}{2} \rho_{11}^{2}\left(x_{1}\left(t-T_{1}(t)\right)\right)-\frac{1}{\rho_{1}} \widetilde{\Phi}_{1} \dot{\hat{\widehat{\omega}}}_{1} \text {, }
\end{aligned}
$$

where

$$
\frac{z_{1}}{k_{b 1}^{2}-z_{1}^{2}} \bar{F}_{1}\left(Z_{1}\right)=\frac{z_{1}}{k_{b 1}^{2}-z_{1}^{2}}\left[W_{1}^{T} S_{1}\left(Z_{1}\right)+\varepsilon_{1}\left(Z_{1}\right)\right]
$$

$$
\begin{aligned}
\bar{F}_{1}\left(Z_{1}\right)= & f_{1}(x)+\frac{z_{1}}{k_{b 1}^{2}-z_{1}^{2}}\left[\varphi_{11}\left(\left\|\bar{x}_{1}\right\|\right)+\varphi_{12}\left(\bar{\alpha}_{1}^{-1}\left(r+D_{0}\right)\right)\right]^{2} \\
& +\frac{z_{1}}{2\left(k_{b 1}^{2}-z_{1}^{2}\right)-\dot{y}_{r}} .
\end{aligned}
$$$$
\leq \frac{\left|z_{1}\right|}{k_{b 1}^{2}-z_{1}^{2}}\left\|W_{1}\right\|\left\|S_{1}\left(Z_{1}\right)\right\|+\frac{\varepsilon_{1}\left(Z_{1}\right) z_{1}}{k_{b 1}^{2}-z_{1}^{2}},
$$

Note that $\bar{F}_{1}\left(Z_{1}\right)$ is an unknown continuous function and RBFNN can be used to approximate it. Hence, from (2), the following equation holds:

$$
\bar{F}_{1}\left(Z_{1}\right)=W_{1}^{T} S_{1}\left(Z_{1}\right)+\varepsilon_{1}\left(Z_{1}\right),
$$

where $\quad W_{1}^{T} S_{1}\left(Z_{1}\right) \quad$ is an $\mathrm{NN}$, $\left|\varepsilon_{1}\left(Z_{1}\right)\right| \leq \varepsilon_{1}, Z_{1}=\left[\bar{x}_{n}, z_{1}, r, \dot{y}_{r}\right]^{T}$, and $\varepsilon_{1}>0$ is any given. $\leq \frac{\left|z_{1}\right|}{k_{b 1}^{2}-z_{1}^{2}}\left\|W_{1}\right\|\left\|S_{1}\left(\Xi_{1}\right)\right\|+\frac{\varepsilon_{1}\left(Z_{1}\right) z_{1}}{k_{b 1}^{2}-z_{1}^{2}}$, $\leq \frac{1}{2 l_{1}} \frac{z_{1}^{2}}{\left(k_{b 1}^{2}-z_{1}^{2}\right)^{2}}\left\|W_{1}\right\|\left\|^{2}\right\| S_{1}\left(\Xi_{1}\right) \|^{2}+\frac{l_{1}}{2}$ $+\frac{\varepsilon_{1} z_{1}}{k_{b 1}^{2}-z_{1}^{2}}$

By using Young's inequality and Lemma 2, one has $=\frac{1}{2 l_{1}} \frac{z_{1}^{2}}{\left(k_{b 1}^{2}-z_{1}^{2}\right)^{2}} \Phi_{1}\left\|S_{1}\left(\Xi_{1}\right)\right\|^{2}+\frac{l_{1}}{2}+\frac{\varepsilon_{1} z_{1}}{k_{b 1}^{2}-z_{1}^{2}}$, 


$$
\begin{aligned}
& \dot{V}_{z_{1}} \leq \frac{g_{1}\left(\bar{x}_{1}\right) z_{1} z_{2}}{k_{b 1}^{2}-z_{1}^{2}}+\frac{g_{1}\left(\bar{x}_{1}\right) z_{1} y_{2}}{k_{b 1}^{2}-z_{1}^{2}}-\frac{c_{1} g_{1}\left(\bar{x}_{1}\right) z_{1}^{2}}{k_{b 1}^{2}-z_{1}^{2}}-\frac{\mu_{1} g_{1}\left(\bar{x}_{1}\right) z_{1}^{2}}{\left(k_{b 1}^{2}-z_{1}^{2}\right)^{2}}-\frac{g_{1}\left(\bar{x}_{1}\right) z_{1}^{2} \widehat{\mathrm{\omega}}_{1}\left\|S_{1}\left(\Xi_{1}\right)\right\|^{2}}{2 l_{1}\left(k_{b 1}^{2}-z_{1}^{2}\right)^{2}}+\frac{l_{1}}{2}, \\
& -\frac{\kappa_{1} g_{1}\left(\bar{x}_{1}\right) z_{1} \beta_{1}\left(z_{1}\right)}{k_{b 1}^{2}-z_{1}^{2}}+\frac{z_{1}^{2} \varpi_{1}\left\|S_{1}\left(\Xi_{1}\right)\right\|^{2}}{2 l_{1}\left(k_{b 1}^{2}-z_{1}^{2}\right)^{2}}+\frac{1}{4}+\frac{\varepsilon_{1} z_{1}}{k_{b 1}^{2}-z_{1}^{2}}+\frac{1}{2} \rho_{11}^{2}\left(x_{1}\left(t-T_{1}(t)\right)\right), \\
& -\frac{1}{\rho_{1}} \widetilde{\mathrm{\omega}}_{1}\left[\rho_{1}\left(-\sigma_{11} \widehat{\widehat{\omega}}_{1}-\sigma_{12} \widehat{\mathrm{\omega}}_{1}^{h}+\frac{z_{1}^{2} S_{1}^{T}\left(\Xi_{1}\right) S_{1}\left(\Xi_{1}\right)}{2 l_{1}\left(k_{b 1}^{2}-z_{1}^{2}\right)^{2}}\right)\right] \text {, } \\
& \leq \frac{g_{1}\left(\bar{x}_{1}\right) z_{1} z_{2}}{k_{b 1}^{2}-z_{1}^{2}}+\frac{g_{1}\left(\bar{x}_{1}\right) z_{1} y_{2}}{k_{b 1}^{2}-z_{1}^{2}}-\frac{b_{1} c_{1} z_{1}^{2}}{k_{b 1}^{2}-z_{1}^{2}}-\frac{\mu_{1} b_{1} z_{1}^{2}}{\left(k_{b 1}^{2}-z_{1}^{2}\right)^{2}}-\frac{z_{1}^{2} \varpi_{1}\left\|S_{1}\left(\Xi_{1}\right)\right\|^{2}-z_{1}^{2} \widetilde{\varpi}_{1}\left\|S_{1}\left(\Xi_{1}\right)\right\|^{2}}{2 l_{1}\left(k_{b 1}^{2}-z_{1}^{2}\right)^{2}}, \\
& +\frac{l_{1}}{2}-\frac{\kappa_{1} g_{1}\left(\bar{x}_{1}\right) z_{1} \beta_{1}\left(z_{1}\right)}{k_{b 1}^{2}-z_{1}^{2}}+\frac{z_{1}^{2} \varpi_{1}\left\|S_{1}\left(\Xi_{1}\right)\right\|^{2}}{2 l_{1}\left(k_{b 1}^{2}-z_{1}^{2}\right)^{2}}+\frac{1}{4}+\frac{\varepsilon_{1} z_{1}}{k_{b 1}^{2}-z_{1}^{2}}+\frac{1}{2} \rho_{11}^{2}\left(x_{1}\left(t-T_{1}(t)\right)\right), \\
& +\sigma_{11} \widetilde{\mathrm{\omega}}_{1} \widehat{\mathrm{\omega}}_{1}+\sigma_{12} \widetilde{\mathrm{\omega}}_{1} \widehat{\mathrm{\omega}}_{1}^{h}-\frac{z_{1}^{2} \widetilde{\mathrm{\omega}}_{1} S_{1}^{T}\left(\Xi_{1}\right) S_{1}\left(\Xi_{1}\right)}{2 l_{1}\left(k_{b 1}^{2}-z_{1}^{2}\right)^{2}}, \\
& \leq \frac{g_{1}\left(\bar{x}_{1}\right) z_{1} z_{2}}{k_{b 1}^{2}-z_{1}^{2}}+\frac{g_{1}\left(\bar{x}_{1}\right) z_{1} y_{2}}{k_{b 1}^{2}-z_{1}^{2}}-\frac{b_{1} c_{1} z_{1}^{2}}{k_{b 1}^{2}-z_{1}^{2}}-\frac{\mu_{1} b_{1} z_{1}^{2}}{\left(k_{b 1}^{2}-z_{1}^{2}\right)^{2}}+\frac{l_{1}}{2}-\frac{\kappa_{1} g_{1}\left(\bar{x}_{1}\right) z_{1} \beta_{1}\left(z_{1}\right)}{k_{b 1}^{2}-z_{1}^{2}}+\frac{1}{4}, \\
& +\frac{\varepsilon_{1} z_{1}}{k_{b 1}^{2}-z_{1}^{2}}+\frac{1}{2} \rho_{11}^{2}\left(x_{1}\left(t-T_{1}(t)\right)\right)+\sigma_{11} \widetilde{\Phi}_{1} \widehat{\varpi}_{1}+\sigma_{12} \widetilde{\varpi}_{1} \widehat{\varpi}_{1}^{h} .
\end{aligned}
$$

By utilizing Young's inequality, the following inequalities can be obtained:

$$
\begin{aligned}
\frac{g_{1}\left(\bar{x}_{1}\right) z_{1} y_{2}}{k_{b 1}^{2}-z_{1}^{2}}-\frac{\mu_{1} b_{1} z_{1}^{2}}{2\left(k_{b 1}^{2}-z_{1}^{2}\right)^{2}} \leq \frac{g_{1}^{2}\left(\bar{x}_{1}\right) y_{2}^{2}}{2 \mu_{1} b_{1}} \leq \frac{a_{1}^{2} y_{2}^{2}}{2 \mu_{1} b_{1}}, \\
\frac{\varepsilon_{1} z_{1}}{k_{b 1}^{2}-z_{1}^{2}}-\frac{\mu_{1} b_{1} z_{1}^{2}}{2\left(k_{b 1}^{2}-z_{1}^{2}\right)^{2}} \leq \frac{\varepsilon_{1}^{2}}{2 \mu_{1} b_{1}} .
\end{aligned}
$$

Therefore, we have

$$
\begin{aligned}
\dot{V}_{z_{1}} \leq & \frac{g_{1}\left(\bar{x}_{1}\right) z_{1} z_{2}}{k_{b 1}^{2}-z_{1}^{2}}+\frac{a_{1}^{2} y_{2}^{2}}{2 \mu_{1} b_{1}}+\frac{\varepsilon_{1}^{2}}{2 \mu_{1} b_{1}}-\frac{b_{1} c_{1} z_{1}^{2}}{k_{b 1}^{2}-z_{1}^{2}}+\frac{l_{1}}{2} \\
& -\frac{\kappa_{1} g_{1}\left(\bar{x}_{1}\right) z_{1} \beta_{1}\left(z_{1}\right)}{k_{b 1}^{2}-z_{1}^{2}}+\frac{1}{4}, \\
& +\frac{1}{2} \rho_{11}^{2}\left(x_{1}\left(t-T_{1}(t)\right)\right)+\sigma_{11} \widetilde{\mathrm{\Phi}}_{1} \widehat{\mathrm{\omega}}_{1}+\sigma_{12} \widetilde{\mathrm{\omega}}_{1} \widehat{\mathrm{\omega}}_{1}^{h} .
\end{aligned}
$$

According to the inequality $2 b_{1} \widetilde{\mathrm{\omega}}_{1} \widehat{\mathrm{\omega}}_{1} \leq \Phi_{1}^{2}-\widetilde{\omega}_{1}^{2}$ and Lemma 4 , one as

$$
\dot{V}_{z_{1}} \leq \frac{g_{1}\left(\bar{x}_{1}\right) z_{1} z_{2}}{k_{b 1}^{2}-z_{1}^{2}}+\frac{a_{1}^{2} y_{2}^{2}}{2 \mu_{1} b_{1}}+\frac{\varepsilon_{1}^{2}}{2 \mu_{1} b_{1}}-\frac{b_{1} c_{1} z_{1}^{2}}{k_{b 1}^{2}-z_{1}^{2}}+\frac{l_{1}}{2}
$$

$$
\begin{aligned}
& -\frac{\kappa_{1} g_{1}\left(\bar{x}_{1}\right) z_{1} \beta_{1}\left(z_{1}\right)}{k_{b 1}^{2}-z_{1}^{2}}+\frac{1}{4}, \\
& +\frac{1}{2} \rho_{11}^{2}\left(x_{1}\left(t-T_{1}(t)\right)\right)+\frac{\sigma_{11}}{2 b_{1}} \varrho_{1}^{2}-\frac{\sigma_{11}}{2 b_{1}} \widetilde{\omega}_{1}^{2}
\end{aligned}
$$

$$
-\frac{\sigma_{12} \zeta_{1}}{b_{1}^{h}} \widetilde{\omega}_{1}^{1+h}+\frac{\sigma_{12} \zeta_{2}}{b_{1}^{h}} \widehat{\omega}_{1}^{1+h},
$$

where $\zeta_{1}$ and $\zeta_{2}$ are defined in Lemma 4.

To deal with the time delay in equation (24), define the Lyapunov-Krasovskii functional as follows:

$$
V_{U_{1}}=\frac{e^{-\gamma\left(t-T_{\max }\right)}}{2\left(1-\bar{T}_{\max }\right)} \int_{t-T_{1}(t)}^{t} e^{\gamma s} \rho_{11}^{2}\left(x_{1}(s)\right) \mathrm{d} s,
$$

where $\gamma>0$ is a positive constant. Using Assumption 5, we obtain that the derivative of $V_{U_{1}}$ is 


$$
\begin{aligned}
\dot{V}_{U_{1}}= & \frac{e^{-\gamma\left(t-T_{\max }\right)}}{2\left(1-\bar{T}_{\max }\right)}\left[e^{\gamma t} \rho_{11}^{2}\left(x_{1}(t)\right)-e^{\gamma\left(t-T_{1}(t)\right)} \rho_{11}^{2}\right. \\
& \left.\cdot\left(x_{1}\left(t-T_{1}(t)\right)\right)\left(1-\dot{T}_{1}(t)\right)\right]-\gamma V_{U_{1}}, \\
\leq & \frac{e^{\gamma T_{\max }}}{2\left(1-\bar{T}_{\max }\right)} \rho_{11}^{2}\left(x_{1}(t)\right)-\frac{1}{2} \rho_{11}^{2}\left(x_{1}\left(t-T_{1}(t)\right)\right)-\gamma V_{U_{1}} .
\end{aligned}
$$

From equations (24) and (26), we have

$$
\begin{aligned}
& \dot{V}_{z_{1}}+\dot{V}_{U_{1}} \leq \frac{g_{1}\left(\bar{x}_{1}\right) z_{1} z_{2}}{k_{b 1}^{2}-z_{1}^{2}}+\frac{a_{1}^{2} y_{2}^{2}}{2 \mu_{1} b_{1}}+\frac{\varepsilon_{1}^{2}}{2 \mu_{1} b_{1}}-\frac{b_{1} c_{1} z_{1}^{2}}{k_{b 1}^{2}-z_{1}^{2}}+\frac{l_{1}}{2} \\
& -\frac{\kappa_{1} g_{1}\left(\bar{x}_{1}\right) z_{1} \beta_{1}\left(z_{1}\right)}{k_{b 1}^{2}-z_{1}^{2}}+\frac{1}{4} \\
& +\frac{\sigma_{11}}{2 b_{1}}{\Theta_{1}^{2}}^{2}-\frac{\sigma_{11}}{2 b_{1}} \widetilde{\Theta}_{1}^{2}-\frac{\sigma_{12} \zeta_{1}}{b_{1}^{h}} \widetilde{\Theta}_{1}^{1+h}+\frac{\sigma_{12} \zeta_{2}}{b_{1}^{h}}{\omega_{1}^{1+h}}^{1+h} \\
& +\Phi_{1}-\gamma V_{U_{1}},
\end{aligned}
$$

where $\Phi_{1}=\left(e^{\gamma T_{\max }} / 2\left(1-\bar{T}_{\max }\right)\right) \rho_{11}^{2}\left(x_{1}(t)\right)$.

To move on, introduce the coordinate transformation

$$
\begin{aligned}
& z_{i}=x_{i}-w_{i}, \\
& y_{i}=w_{i}-\alpha_{i-1},
\end{aligned}
$$

where $z_{i}, \alpha_{i-1}$, and $y_{i}$ denote the tracking error, the virtual control input, and the boundary layer error for $i=2,3, \ldots, n$, respectively. $w_{i}$ is the output of the following first-order filter:

$$
\dot{w}_{i}=-\tau_{i 1} y_{i}-\tau_{i 2} y_{i}^{h}
$$

where $\tau_{i 1}$ and $\tau_{i 2}$ are the positive design constants and $h$ is defined in (15).

Remark 3. From (29), it can be seen that the proposed filter involves both the linear and fractional terms. In particular, when $\tau_{i 1}=0$ or $\tau_{i 2}=0$, filter (29) degrades into the fractional filter used in [58] and the linear filter as widely used in the literature [21-23], respectively. It is the key to ensure the fast finite-time stability of the closed-loop system, which will be detailed in the following analysis.

Remark 4. As mentioned in [34], by designing $\iota_{11}$ and $\iota_{12}$ properly, both the virtual control input $\alpha_{1}$ and its derivative $\dot{\alpha}_{1}$ are ensured to be inherently continuous in the set $\Omega_{x_{i}}$. It means that the virtual control input $\alpha_{1}$ defined in (13) is $C^{1}$ continuous in the set $\Omega_{x_{i}}$. From (13), it is not hard to see that $\alpha_{1}$ and its derivative $\dot{\alpha}_{1}$ are the functions of the variables $z_{1}, \widehat{\omega}_{1}, \dot{y}_{r}$ and $z_{1}, z_{2}, \dot{\widehat{\omega}}_{1}, y_{2}, \dot{y}_{r}, \ddot{y}_{r}$, respectively. Combining the continuity of $\dot{\alpha}_{1}$ and (28) and (29), it can be seen that there exists a continuous function $\lambda_{2}\left(z_{1}, z_{2}, \dot{\widehat{\omega}}_{1}, y_{2}, \dot{y}_{r}, \ddot{y}_{r}\right)$ which satisfies

$$
\dot{y}_{2} \leq-\tau_{21} y_{2}-\tau_{22} y_{2}^{h}+\lambda_{2}\left(z_{1}, z_{2}, \dot{\widehat{\omega}}_{1}, y_{2}, \dot{y}_{r}, \ddot{y}_{r}\right)
$$

Step 2. $(i=2,3, \ldots, n-1)$ Define the $i^{\text {th }}$ surface error $z_{i}=x_{i}-w_{i}$; the time derivative of $z_{i}$ is defined as

$$
\begin{aligned}
\dot{z}_{i}= & f_{i}(x)+g_{i}\left(\bar{x}_{i}\right) x_{i+1}+\delta_{i}(\xi, x, t)+d_{i}\left(\bar{x}_{i}\left(t-T_{i}(t)\right)\right)-\dot{w}_{i}, \\
= & f_{i}(x)+g_{i}\left(\bar{x}_{i}\right)\left(z_{i+1}+y_{i+1}+\alpha_{i}\right)+\delta_{i}(\xi, x, t) \\
& +d_{i}\left(\bar{x}_{i}\left(t-T_{i}(t)\right)\right)-\dot{w}_{i} .
\end{aligned}
$$

The virtual control law $\alpha_{i}$ and the update law $\dot{\widehat{\widehat{\omega}}}_{i}$ are designed as

$$
\begin{aligned}
& \alpha_{i}=-c_{i} z_{i}-\mu_{i} \frac{z_{i}}{k_{b i}^{2}-z_{i}^{2}}-\frac{z_{i} \widehat{\mathrm{\omega}}_{i} S_{i}^{T}\left(\Xi_{i}\right) S_{i}\left(\Xi_{i}\right)}{2 l_{i}\left(k_{b i}^{2}-z_{i}^{2}\right)}-\kappa_{i} \beta_{i}\left(z_{i}\right), \\
& \dot{\widehat{\widehat{\omega}}}_{i}=\rho_{i}\left(-\sigma_{i 1} \widehat{\widehat{\omega}}_{i}-\sigma_{i 2} \widehat{\oplus}_{i}^{h}+\frac{z_{i}^{2} S_{i}^{T}\left(\Xi_{i}\right) S_{i}\left(\Xi_{i}\right)}{2 l_{i}\left(k_{b i}^{2}-z_{i}^{2}\right)^{2}}\right),
\end{aligned}
$$

where $c_{i}, \mu_{i}, k_{b i}, \kappa_{i}, \rho_{i}, \sigma_{i 1}, \sigma_{i 2}, l_{i}$ are positive design parame-

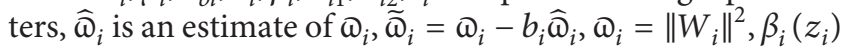
is defined as

$$
\beta_{i}\left(z_{i}\right)= \begin{cases}z_{i}^{h}\left(k_{b i}^{2}-z_{i}^{2}\right)^{(1-h / 2)}, & \text { if }\left|z_{i}\right| \geq \tau_{i}, \\ \iota_{i 1} z_{i}+\iota_{i 2} z_{i}^{3}, & \text { if }\left|z_{i}\right|<\tau_{i}\end{cases}
$$

where $h$ is defined in (15), $\iota_{i 1}=\tau_{i}^{h-1}\left(k_{b i}^{2}-\tau_{i}^{2}\right)^{1-h / 2}-\iota_{i 2} \tau_{i}^{2}$, $\iota_{i 2}=\left(1 / 2 \tau_{i}^{3}\right)(h-1) \tau_{i}^{h}\left[\left(k_{b i}^{2}-\tau_{i}^{2}\right)^{(1-h / 2)}+\tau_{i}^{2}\left(\left(k_{b i}^{2}-\tau_{i}^{2}\right)^{-(1+h / 2)}\right)\right]$, and $\tau_{i}<k_{b i}$ is a small positive constant.

Consider the BLF candidate $V_{z_{i}}$ as

$$
V_{z_{i}}=\frac{1}{2} \log \frac{k_{b i}^{2}}{k_{b i}^{2}-z_{i}^{2}}+\frac{1}{2 b_{i} \rho_{i}} \widetilde{\widetilde{\omega}}_{i}^{2},
$$

where $V_{z_{i}}$ is also positive definite and continuously differentiable in the set $\left|z_{i}\right|<k_{b i}$. Similar to (17), the time derivative of $V_{z_{i}}$ is

$$
\begin{aligned}
\dot{V}_{z_{i}} \leq & \frac{z_{i}}{k_{b i}^{2}-z_{i}^{2}}\left[\bar{F}_{i}\left(Z_{i}\right)+g_{i}\left(\bar{x}_{i}\right)\left(z_{i+1}+y_{i+1}+\alpha_{i}\right)\right] \\
& +\frac{1}{4}-\frac{1}{\rho_{i}} \widetilde{\omega}_{i} \dot{\hat{\mathrm{\omega}}}_{i}, \\
& +\frac{1}{2} \sum_{j=1}^{i} \rho_{i j}^{2}\left(x_{j}\left(t-T_{j}(t)\right)\right)-\frac{g_{i-1}\left(\bar{x}_{i-1}\right) z_{i-1} z_{i}}{k_{b(i-1)}^{2}-z_{i-1}^{2}},
\end{aligned}
$$

where

$$
\begin{aligned}
\bar{F}_{i}\left(Z_{i}\right)= & f_{i}(x)+\frac{z_{i}}{k_{b i}^{2}-z_{i}^{2}}\left[\varphi_{i 1}\left(\left\|\bar{x}_{i}\right\|\right)+\varphi_{i 2}\left(\bar{\alpha}_{i}^{-1}\left(r+D_{0}\right)\right)\right]^{2} \\
& -\dot{w}_{i}+\frac{g_{i-1}\left(\bar{x}_{i-1}\right)\left(k_{b i}^{2}-z_{i}^{2}\right) z_{i-1}}{k_{b(i-1)}^{2}-z_{i-1}^{2}}+\frac{i z_{i}}{2\left(k_{b i}^{2}-z_{i}^{2}\right)} .
\end{aligned}
$$


Note that $\bar{F}_{i}\left(Z_{i}\right)$ is an unknown continuous function and RBFNN can be used to approximate it. Hence, from (2), the following equation holds:

$$
\bar{F}_{i}\left(Z_{i}\right)=W_{i}^{T} S_{i}\left(Z_{i}\right)+\varepsilon_{i}\left(Z_{i}\right)
$$

where $\quad W_{i}^{T} S_{i}\left(Z_{i}\right) \quad$ is an $\mathrm{NN}, \quad\left|\varepsilon_{i}\left(Z_{i}\right)\right| \leq \varepsilon_{i}, Z_{i}=$ $\left[\bar{x}_{n}, z_{i-1}, z_{i}, r, w_{i-1}, \dot{w}_{i}\right]^{T}$, and $\varepsilon_{i}>0$ is any given.

By using Young's inequality and Lemma 2, one has

$$
\begin{aligned}
\frac{z_{i}}{k_{b i}^{2}-z_{i}^{2}} \bar{F}_{i}\left(Z_{i}\right) & =\frac{z_{i}}{k_{b i}^{2}-z_{i}^{2}}\left[W_{i}^{T} S_{i}\left(Z_{i}\right)+\varepsilon_{i}\left(Z_{i}\right)\right] \\
& \leq \frac{\left|z_{i}\right|}{k_{b i}^{2}-z_{i}^{2}}\left\|W_{i}\right\|\left\|S_{i}\left(Z_{i}\right)\right\|+\frac{\varepsilon_{i}\left(Z_{i}\right) z_{i}}{k_{b i}^{2}-z_{i}^{2}}, \\
& \leq \frac{\left|z_{i}\right|}{k_{b i}^{2}-z_{i}^{2}}\left\|W_{i}\right\|\left\|S_{i}\left(\Xi_{i}\right)\right\|+\frac{\varepsilon_{i}\left(Z_{i}\right) z_{i}}{k_{b i}^{2}-z_{i}^{2}}, \\
& \leq \frac{1}{2 l_{i}} \frac{z_{i}^{2}}{\left(k_{b i}^{2}-z_{i}^{2}\right)^{2}}\left\|W_{i}\right\|^{2}\left\|S_{i}\left(\Xi_{i}\right)\right\|^{2}+\frac{l_{i}}{2}+\frac{\varepsilon_{i}\left(Z_{i}\right) z_{i}}{k_{b i}^{2}-z_{i}^{2}}
\end{aligned}
$$

Substituting (32), (33), and (39) into (36), we can obtain

$$
\begin{aligned}
& \dot{V}_{z_{i}} \leq \frac{g_{i}\left(\bar{x}_{i}\right) z_{i} z_{i+1}}{k_{b i}^{2}-z_{i}^{2}}+\frac{g_{i}\left(\bar{x}_{i}\right) z_{i} y_{i+1}}{k_{b i}^{2}-z_{i}^{2}}-\frac{c_{i} g_{i}\left(\bar{x}_{i}\right) z_{i}^{2}}{k_{b i}^{2}-z_{i}^{2}}-\frac{\mu_{i} g_{i}\left(\bar{x}_{i}\right) z_{i}^{2}}{\left(k_{b i}^{2}-z_{i}^{2}\right)^{2}}-\frac{g_{i}\left(\bar{x}_{i}\right) z_{i}^{2} \widehat{\mathrm{\omega}}_{i}\left\|S_{i}\left(\Xi_{i}\right)\right\|^{2}}{2 l_{i}\left(k_{b i}^{2}-z_{i}^{2}\right)^{2}}+\frac{l_{i}}{2}+\frac{1}{4}, \\
& -\frac{\kappa_{i} g_{i}\left(\bar{x}_{i}\right) z_{i} \beta_{i}\left(z_{i}\right)}{k_{b i}^{2}-z_{i}^{2}}+\frac{z_{i}^{2} ळ_{i}\left\|S_{i}\left(\Xi_{i}\right)\right\|^{2}}{2 l_{i}\left(k_{b i}^{2}-z_{i}^{2}\right)^{2}}+\frac{\varepsilon_{i}\left(Z_{i}\right) z_{i}}{k_{b i}^{2}-z_{i}^{2}}+\frac{1}{2} \sum_{j=1}^{i} \rho_{i j}^{2}\left(x_{j}\left(t-T_{j}(t)\right)\right), \\
& -\frac{g_{i-1}\left(\bar{x}_{i-1}\right) z_{i-1} z_{i}}{k_{b(i-1)}^{2}-z_{i-1}^{2}}-\frac{1}{\rho_{i}} \widetilde{\omega}_{i}\left[\rho_{i}\left(-\sigma_{i 1} \widehat{\varpi}_{i}-\sigma_{i 2} \widehat{\widehat{\omega}}_{i}^{h}+\frac{z_{i}^{2} S_{i}^{T}\left(\Xi_{i}\right) S_{i}\left(\Xi_{i}\right)}{2 l_{i}\left(k_{b i}^{2}-z_{i}^{2}\right)^{2}}\right)\right], \\
& \leq \frac{g_{i}\left(\bar{x}_{i}\right) z_{i} z_{i+1}}{k_{b i}^{2}-z_{i}^{2}}+\frac{g_{i}\left(\bar{x}_{i}\right) z_{i} y_{i+1}}{k_{b i}^{2}-z_{i}^{2}}-\frac{b_{i} c_{i} z_{i}^{2}}{k_{b i}^{2}-z_{i}^{2}}-\frac{\mu_{i} b_{i} z_{i}^{2}}{\left(k_{b i}^{2}-z_{i}^{2}\right)^{2}}-\frac{z_{i}^{2} \varpi_{i}\left\|S_{i}\left(\Xi_{i}\right)\right\|^{2}-z_{i}^{2} \widetilde{\omega}_{i}\left\|S_{i}\left(\Xi_{i}\right)\right\|^{2}}{2 l_{i}\left(k_{b i}^{2}-z_{i}^{2}\right)^{2}}, \\
& +\frac{l_{i}}{2}+\frac{1}{4}-\frac{\kappa_{i} g_{i}\left(\bar{x}_{i}\right) z_{i} \beta_{i}\left(z_{i}\right)}{k_{b i}^{2}-z_{i}^{2}}+\frac{z_{i}^{2} \varpi_{i}\left\|S_{i}\left(\Xi_{i}\right)\right\|^{2}}{2 l_{i}\left(k_{b i}^{2}-z_{i}^{2}\right)^{2}}+\frac{\varepsilon_{i}\left(Z_{i}\right) z_{i}}{k_{b i}^{2}-z_{i}^{2}}+\frac{1}{2} \sum_{j=1}^{i} \rho_{i j}^{2}\left(x_{j}\left(t-T_{j}(t)\right)\right), \\
& \left.+\sigma_{i 1} \widetilde{\mathrm{\omega}}_{i} \widehat{\mathrm{\omega}}_{i}+\sigma_{i 2} \widetilde{\mathrm{\omega}}_{i} \widehat{\mathrm{\omega}}_{i}^{h}-\frac{z_{i}^{2} \widetilde{\mathrm{\omega}}_{i} S_{i}^{T}\left(\Xi_{i}\right) S_{i}\left(\Xi_{i}\right)}{2 l_{i}\left(k_{b i}^{2}-z_{i}^{2}\right)^{2}}\right)-\frac{g_{i-1}\left(\bar{x}_{i-1}\right) z_{i-1} z_{i}}{k_{b(i-1)}^{2}-z_{i-1}^{2}}, \\
& \leq \frac{g_{i}\left(\bar{x}_{i}\right) z_{i} z_{i+1}}{k_{b i}^{2}-z_{i}^{2}}+\frac{g_{i}\left(\bar{x}_{i}\right) z_{i} y_{i+1}}{k_{b i}^{2}-z_{i}^{2}}-\frac{b_{i} c_{i} z_{i}^{2}}{k_{b i}^{2}-z_{i}^{2}}-\frac{\mu_{i} b_{i} z_{i}^{2}}{\left(k_{b i}^{2}-z_{i}^{2}\right)^{2}}+\frac{l_{i}}{2}+\frac{1}{4}-\frac{\kappa_{i} g_{i}\left(\bar{x}_{i}\right) z_{i} \beta_{i}\left(z_{i}\right)}{k_{b i}^{2}-z_{i}^{2}}+\frac{\varepsilon_{i}\left(Z_{i}\right) z_{i}}{k_{b i}^{2}-z_{i}^{2}} \\
& +\frac{1}{2} \sum_{j=1}^{i} \rho_{i j}^{2}\left(x_{j}\left(t-T_{j}(t)\right)\right)+\sigma_{i 1} \widetilde{\mathrm{\omega}}_{i} \widehat{\widehat{\omega}}_{i}+\sigma_{i 2} \widetilde{\mathrm{\omega}}_{i} \widehat{\mathrm{\omega}}_{i}^{h}-\frac{g_{i-1}\left(\bar{x}_{i-1}\right) z_{i-1} z_{i}}{k_{b(i-1)}^{2}-z_{i-1}^{2}} .
\end{aligned}
$$


By utilizing Young's inequality, the following inequalities can be obtained:

$$
\begin{gathered}
\frac{g_{i}\left(\bar{x}_{i}\right) z_{i} y_{i+1}}{k_{b i}^{2}-z_{i}^{2}}-\frac{\mu_{i} b_{i} z_{i}^{2}}{2\left(k_{b i}^{2}-z_{i}^{2}\right)^{2}} \leq \frac{g_{i}^{2}\left(\bar{x}_{i}\right) y_{i+1}^{2}}{2 \mu_{i} b_{i}} \leq \frac{a_{i}^{2} y_{i+1}^{2}}{2 \mu_{i} b_{i}}, \\
\frac{\varepsilon_{i}\left(Z_{i}\right) z_{i}}{k_{b i}^{2}-z_{i}^{2}}-\frac{\mu_{i} b_{i} z_{i}^{2}}{2\left(k_{b i}^{2}-z_{i}^{2}\right)^{2}} \leq \frac{\varepsilon_{i}^{2}\left(Z_{i}\right)}{2 \mu_{i} b_{i}} \leq \frac{\varepsilon_{i}^{2}}{2 \mu_{i} b_{i}} .
\end{gathered}
$$

Therefore, we have

$$
\begin{aligned}
\dot{V}_{z_{i}} \leq & \frac{g_{i}\left(\bar{x}_{i}\right) z_{i} z_{i+1}}{k_{b i}^{2}-z_{i}^{2}}+\frac{a_{i}^{2} y_{i+1}^{2}}{2 \mu_{i} b_{i}}+\frac{\varepsilon_{i}^{2}}{2 \mu_{i} b_{i}}-\frac{b_{i} c_{i} z_{i}^{2}}{k_{b i}^{2}-z_{i}^{2}} \\
& -\frac{\kappa_{i} g_{i}\left(\bar{x}_{i}\right) z_{i} \beta_{i}\left(z_{i}\right)}{k_{b i}^{2}-z_{i}^{2}}, \\
& +\frac{1}{2} \sum_{j=1}^{i} \rho_{i j}^{2}\left(x_{j}\left(t-T_{j}(t)\right)\right)+\frac{l_{i}}{2}+\frac{1}{4}+\sigma_{i 1} \widetilde{\mathrm{\omega}}_{i} \widehat{\mathrm{\omega}}_{i}+\sigma_{i 2} \widetilde{\mathrm{\omega}}_{i} \widehat{\mathrm{\omega}}_{i}^{h} \\
& -\frac{g_{i-1}\left(\bar{x}_{i-1}\right) z_{i-1} z_{i}}{k_{b(i-1)}^{2}-z_{i-1}^{2}} .
\end{aligned}
$$

According to the inequality $2 b_{i} \widetilde{\Phi}_{i} \widehat{\Phi}_{i} \leq \varpi_{i}^{2}-\widetilde{\Phi}_{i}^{2}$ and Lemma 4, one has

$$
\begin{aligned}
\dot{V}_{z_{i}} \leq & \frac{g_{i}\left(\bar{x}_{i}\right) z_{i} z_{i+1}}{k_{b i}^{2}-z_{i}^{2}}+\frac{a_{i}^{2} y_{i+1}^{2}}{2 \mu_{i} b_{i}}+\frac{\varepsilon_{i}^{2}}{2 \mu_{i} b_{i}}-\frac{b_{i} c_{i} z_{i}^{2}}{k_{b i}^{2}-z_{i}^{2}} \\
& -\frac{\kappa_{i} g_{i}\left(\bar{x}_{i}\right) z_{i} \beta\left(z_{i}\right)}{k_{b i}^{2}-z_{i}^{2}}, \\
& +\frac{1}{2} \sum_{j=1}^{i} \rho_{i j}^{2}\left(x_{j}\left(t-T_{j}(t)\right)\right)+\frac{l_{i}}{2}+\frac{1}{4}+\frac{\sigma_{i 1}}{2 b_{i}} \widehat{\omega}_{i}^{2}-\frac{\sigma_{i 1}}{2 b_{i}} \widetilde{\omega}_{i}^{2} \\
& -\frac{\sigma_{i 2} \zeta_{1}}{b_{i}^{h}} \widetilde{\omega}_{i}^{1+h}+\frac{\sigma_{i 2} \zeta_{2}}{b_{i}^{h}}{\omega_{i}^{1+h}}^{1+h} \frac{g_{i-1}\left(\bar{x}_{i-1}\right) z_{i-1} z_{i}}{k_{b(i-1)}^{2}-z_{i-1}^{2}},
\end{aligned}
$$

where $\zeta_{1}$ and $\zeta_{2}$ are defined in Lemma 4.

To handle the time delay, define the Lyapunov-Krasovskii functional as follows:

$$
V_{U_{i}}=\frac{e^{-\gamma\left(t-T_{\max }\right)}}{2\left(1-\bar{T}_{\max }\right)} \sum_{j=1}^{i} \int_{t-T_{j}(t)}^{t} e^{\gamma s} \rho_{i j}^{2}\left(x_{j}(s)\right) \mathrm{d} s,
$$

where $\gamma>0$ is a positive constant. By using Assumption 5, we obtain that the derivative of $V_{U i}$ is

$$
\begin{aligned}
\dot{V}_{U_{i}}= & \frac{e^{-\gamma\left(t-T_{\max }\right)}}{2\left(1-\bar{T}_{\max }\right)} \sum_{j=1}^{i}\left[e^{\gamma t} \rho_{i j}^{2}\left(x_{j}(t)\right)-e^{\gamma\left(t-T_{j}(t)\right)} \rho_{i j}^{2}\right. \\
& \left.\cdot\left(x_{j}\left(t-T_{j}(t)\right)\right)\left(1-\dot{T}_{j}(t)\right)\right]-\gamma V_{U_{i}}, \\
\leq & \sum_{j=1}^{i} \frac{e^{\gamma T_{\max }}}{2\left(1-\bar{T}_{\max }\right)} \rho_{i j}^{2}\left(x_{j}(t)\right)-\frac{1}{2} \sum_{j=1}^{i} \rho_{i j}^{2}\left(x_{j}\left(t-T_{j}(t)\right)\right)-\gamma V_{U_{i}} .
\end{aligned}
$$

From in equations (43) and (45), we have

$$
\begin{aligned}
\dot{V}_{z_{i}}+\dot{V}_{U_{i}} \leq & \frac{g_{i}\left(\bar{x}_{i}\right) z_{i} z_{i+1}}{k_{b i}^{2}-z_{i}^{2}}+\frac{a_{i}^{2} y_{i+1}^{2}}{2 \mu_{i} b_{i}}+\frac{\varepsilon_{i}^{2}}{2 \mu_{i} b_{i}}-\frac{b_{i} c_{i} z_{i}^{2}}{k_{b i}^{2}-z_{i}^{2}} \\
& -\frac{\kappa_{i} g_{i}\left(\bar{x}_{i}\right) z_{i} \beta_{i}\left(z_{i}\right)}{k_{b i}^{2}-z_{i}^{2}}+\frac{l_{i}}{2}+\frac{1}{4}+\frac{\sigma_{i 1}}{2 b_{i}} \oplus_{i}^{2}, \\
& -\frac{\sigma_{i 1}}{2 b_{i}} \widetilde{\Theta}_{i}^{2}-\frac{\sigma_{i 2} \zeta_{1}}{b_{i}^{h}} \widetilde{\varpi}_{i}^{1+h}+\frac{\sigma_{i 2} \zeta_{2}}{b_{i}^{h}}{\omega_{i}^{1+h}}^{1+h} \\
& -\frac{g_{i-1}\left(\bar{x}_{i-1}\right) z_{i-1} z_{i}}{k_{b(i-1)}^{2}-z_{i-1}^{2}}+\Phi_{i}-\gamma V_{U_{i}},
\end{aligned}
$$

where $\Phi_{i}=\sum_{j=1}^{i}\left(e^{\gamma T_{\max }} /\left(2\left(1-\bar{T}_{\max }\right)\right)\right) \rho_{i j}^{2}\left(x_{j}(t)\right)$.

Similar to the analysis in Remark 4 , there exists a continuous function $\lambda_{i+1}\left(\bar{z}_{i+1}, \overline{\widehat{\widehat{\omega}}}_{i}, y_{2}, y_{3}, \backslash \ldots, y_{i+1}, \dot{y}_{r}, \ddot{y}_{r}\right)$ which satisfies

$$
\begin{aligned}
\dot{y}_{i+1} \leq & -\tau_{(i+1) 1} y_{i+1}-\tau_{(i+1) 2} y_{i+1}^{h}+\lambda_{i+1} \\
& \cdot\left(\bar{z}_{i+1}, \overline{\widehat{\hat{\omega}}}_{i}, y_{2}, y_{3}, \ldots, y_{i+1}, \dot{y}_{r}, \ddot{y}_{r}\right) .
\end{aligned}
$$

Step 3. Define the $n^{\text {th }}$ surface error $z_{n}=x_{n}-w_{n}$; the time derivative of $z_{n}$ is defined as

$$
\dot{z}_{n}=f_{n}(x)+g_{n}\left(\bar{x}_{n}\right) u+\delta_{n}(\xi, x, t)+d_{n}\left(\bar{x}_{n}\left(t-T_{n}(t)\right)\right)-\dot{w}_{n} .
$$

The actual control law $u$ and the update law $\dot{\widehat{\widehat{\omega}}}_{n}$ are designed as

$$
u=-c_{n} z_{n}-\mu_{n} \frac{z_{n}}{2\left(k_{b n}^{2}-z_{n}^{2}\right)}-\frac{z_{n} \widehat{\Phi}_{n} S_{n}^{T}\left(\Xi_{n}\right) S_{n}\left(\Xi_{n}\right)}{2 l_{n}\left(k_{b n}^{2}-z_{n}^{2}\right)}-\kappa_{n} \beta_{n}\left(z_{n}\right),
$$

$\dot{\hat{\oplus}}_{n}=\rho_{n}\left(-\sigma_{n 1} \widehat{\widehat{\Phi}}_{n}-\sigma_{n 2} \widehat{\widehat{\omega}}_{n}^{h}+\frac{z_{n}^{2} S_{n}^{T}\left(\Xi_{n}\right) S_{n}\left(\Xi_{n}\right)}{2 l_{n}\left(k_{b n}^{2}-z_{n}^{2}\right)^{2}}\right)$,

where $c_{n}, \mu_{n}, k_{b n}, \kappa_{n}, \rho_{n}, \sigma_{n 1}, \sigma_{n 2}, l_{n}$ are positive design parameters, $\widehat{\varpi}_{n}$ is an estimate of $\varpi_{n}, \widetilde{\varpi}_{n}=\varpi_{n}-b_{n} \widehat{\varpi}_{n}, \varpi_{n}=$ $\left\|W_{n}\right\|^{2}, \beta_{n}\left(z_{n}\right)$ is defined as

$$
\beta_{n}\left(z_{n}\right)= \begin{cases}z_{n}^{h}\left(k_{b n}^{2}-z_{n}^{2}\right)^{(1-h / 2)}, & \text { if }\left|z_{n}\right| \geq \tau_{n}, \\ \iota_{n 1} z_{n}+\iota_{n 2} z_{n}^{3}, & \text { if }\left|z_{n}\right|<\tau_{n},\end{cases}
$$


10

Complexity

where $h$ is defined in (15), $\iota_{n 1}=\tau_{n}^{h-1}\left(k_{b n}^{2}-\tau_{n}^{2}\right)^{1-h / 2}-\iota_{n 2} \tau_{n}^{2}, \iota_{n 2}=$ $\left(1 / 2 \tau_{n}^{3}\right)(h-1) \tau_{n}^{h}\left[\left(k_{b n}^{2}-\tau_{n}^{2}\right)^{1-h / 2}+\tau_{n}^{2}\left(\left(k_{b n}^{2}-\tau_{n}^{2}\right)^{-(1+h / 2)}\right)\right]$, and $\tau_{n}<k_{b n}$ is a small positive constant.

Consider the BLF candidate $V_{z_{n}}$ as

$$
V_{z_{n}}=\frac{1}{2} \log \frac{k_{b n}^{2}}{k_{b n}^{2}-z_{n}^{2}}+\frac{1}{2 b_{n} \rho_{n}} \widetilde{\Phi}_{n}^{2} .
$$

Similar to (17) and (36), we can obtain the time deprivative of $V_{z_{n}}$ as follows:

$$
\begin{aligned}
\dot{V}_{z_{n}} \leq & \frac{z_{n}}{k_{b n}^{2}-z_{n}^{2}}\left[\bar{F}_{n}\left(Z_{n}\right)+g_{n}\left(\bar{x}_{n}\right) u\right]+\frac{1}{4}+\frac{1}{2} \sum_{j=1}^{n} \rho_{n j}^{2} \\
& \cdot\left(x_{j}\left(t-T_{j}(t)\right)\right)-\frac{1}{\rho_{n}} \widetilde{\widetilde{\omega}}_{n} \dot{\hat{\widehat{\omega}}}_{n}-\frac{g_{n-1}\left(\bar{x}_{n-1}\right) z_{n-1} z_{n}}{k_{b(n-1)}^{2}-z_{n-1}^{2}},
\end{aligned}
$$

where

$$
\begin{aligned}
\bar{F}_{n}\left(Z_{n}\right)= & f_{n}(x)+\frac{z_{n}}{k_{b n}^{2}-z_{n}^{2}}\left[\varphi_{n 1}\left(\left\|\bar{x}_{n}\right\|\right)+\varphi_{n 2}\left(\bar{\alpha}_{n}^{-1}\left(r+D_{0}\right)\right)\right]^{2}-\dot{w}_{n}, \\
& +\frac{g_{n-1}\left(\bar{x}_{n-1}\right)\left(k_{b n}^{2}-z_{n}^{2}\right) z_{n-1}}{k_{b(n-1)}^{2}-z_{n-1}^{2}}+\frac{n z_{n}}{2\left(k_{b n}^{2}-z_{n}^{2}\right)} .
\end{aligned}
$$

Note that $\bar{F}_{n}\left(Z_{n}\right)$ is an unknown continuous function and RBFNN can be used to approximate it. Hence, from (2), the following equation holds:

$$
\bar{F}_{n}\left(Z_{n}\right)=W_{n}^{T} S_{n}\left(Z_{n}\right)+\varepsilon_{n}\left(Z_{n}\right)
$$

where $\quad W_{n}^{T} S_{n}\left(Z_{n}\right) \quad$ is an $\mathrm{NN}, \quad\left|\varepsilon_{n}\left(Z_{n}\right)\right| \leq \varepsilon_{n}, Z_{n}=$ $\left[\bar{x}_{n}, z_{n-1}, z_{n}, r, w_{n-1}, \dot{w}_{n}\right]^{T}$, and $\varepsilon_{n}>0$ is any given.

By using Young's inequality and Lemma 2, one has

$$
\begin{aligned}
\frac{z_{n}}{k_{b n}^{2}-z_{n}^{2}} \bar{F}_{n}\left(Z_{n}\right)= & \frac{z_{n}}{k_{b n}^{2}-z_{n}^{2}}\left[W_{n}^{T} S_{n}\left(Z_{n}\right)+\varepsilon_{n}\left(Z_{n}\right)\right], \\
\leq & \frac{\left|z_{n}\right|}{k_{b n}^{2}-z_{n}^{2}}\left\|W_{n}\right\|\left\|S_{n}\left(Z_{n}\right)\right\|+\frac{\varepsilon_{n}\left(Z_{n}\right) z_{n}}{k_{b n}^{2}-z_{n}^{2}} \\
\leq & \frac{\left|z_{n}\right|}{k_{b n}^{2}-z_{n}^{2}}\left\|W_{n}\right\|\left\|S_{n}\left(\Xi_{n}\right)\right\|+\frac{\varepsilon_{n}\left(Z_{n}\right) z_{n}}{k_{b n}^{2}-z_{n}^{2}} \\
\leq & \frac{1}{2 l_{n}} \frac{z_{n}^{2}}{\left(k_{b n}^{2}-z_{n}^{2}\right)^{2}}\left\|W_{n}\right\|^{2}\left\|S_{n}\left(\Xi_{n}\right)\right\|^{2}+\frac{l_{n}}{2} \\
& +\frac{\varepsilon_{n}\left(Z_{n}\right) z_{n}}{k_{b n}^{2}-z_{n}^{2}}, \\
= & \frac{1}{2 l_{n}} \frac{z_{n}^{2}}{\left(k_{b n}^{2}-z_{n}^{2}\right)^{2}} \Phi_{n}\left\|S_{n}\left(\Xi_{n}\right)\right\|^{2} \\
& +\frac{l_{n}}{2}+\frac{\varepsilon_{n}\left(Z_{n}\right) z_{n}}{k_{b n}^{2}-z_{n}^{2}},
\end{aligned}
$$

where $\Xi_{n}=Z_{n}=\left[\bar{x}_{n}, z_{n-1}, z_{n}, r, w_{n-1}, \dot{w}_{n}\right]^{T}$.

Substituting (49), (50), and (56) into (53), we can obtain

$$
\begin{aligned}
\dot{V}_{z_{n}} \leq & -\frac{b_{n} c_{n} z_{n}^{2}}{k_{b n}^{2}-z_{n}^{2}}-\frac{\mu_{n} b_{n} z_{n}^{2}}{2\left(k_{b n}^{2}-z_{n}^{2}\right)^{2}}-\frac{\kappa_{n} g_{n}\left(\bar{x}_{n}\right) z_{n} \beta_{n}\left(z_{n}\right)}{k_{b n}^{2}-z_{n}^{2}} \\
& +\frac{l_{n}}{2}+\frac{1}{4}+\frac{\varepsilon_{n}\left(Z_{n}\right) z_{n}}{k_{b n}^{2}-z_{n}^{2}} \\
& +\frac{1}{2} \sum_{j=1}^{n} \rho_{n j}^{2}\left(x_{j}\left(t-T_{j}(t)\right)\right)+\sigma_{n 1}{\widetilde{\omega_{n}}}_{n} \widehat{\mathrm{\omega}}_{n}+\sigma_{n 2} \widetilde{\mathrm{\omega}}_{n} \widehat{\mathrm{\omega}}_{n}^{h} \\
& -\frac{g_{n-1}\left(\bar{x}_{n-1}\right) z_{n-1} z_{n}}{k_{b(n-1)}^{2}-z_{n-1}^{2}} .
\end{aligned}
$$

By utilizing Young's inequality, the following inequality can be obtained:

$$
\frac{\varepsilon_{n}\left(Z_{n}\right) z_{n}}{k_{b n}^{2}-z_{n}^{2}}-\frac{\mu_{n} b_{n} z_{n}^{2}}{2\left(k_{b n}^{2}-z_{n}^{2}\right)^{2}} \leq \frac{\varepsilon_{n}^{2}\left(Z_{n}\right)}{2 \mu_{n} b_{n}} \leq \frac{\varepsilon_{n}^{2}}{2 \mu_{n} b_{n}} .
$$

Therefore, we have

$$
\begin{aligned}
& \dot{V}_{z_{n}} \leq-\frac{b_{n} c_{n} z_{n}^{2}}{k_{b n}^{2}-z_{n}^{2}}+\frac{\varepsilon_{n}^{2}}{2 \mu_{n} b_{n}}-\frac{\kappa_{n} g_{n}\left(\bar{x}_{n}\right) z_{n} \beta_{n}\left(z_{n}\right)}{k_{b n}^{2}-z_{n}^{2}} \\
& +\frac{l_{n}}{2}+\frac{1}{4}+\frac{1}{2} \sum_{j=1}^{n} \rho_{n j}^{2}\left(x_{j}\left(t-T_{j}(t)\right)\right) \text {, }
\end{aligned}
$$

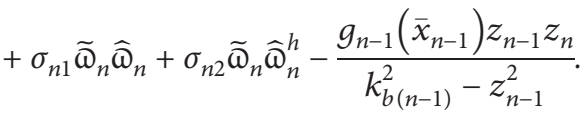

According to the inequality $2 b_{n} \widetilde{\mathrm{\omega}}_{n} \widehat{\mathrm{\omega}}_{n} \leq{\omega_{n}^{2}}^{2}-\widetilde{\mathrm{\omega}}_{n}^{2}$ and Lemma 4, one has

$$
\begin{aligned}
\dot{V}_{z_{n}} \leq & -\frac{b_{n} c_{n} z_{n}^{2}}{k_{b n}^{2}-z_{n}^{2}}+\frac{\varepsilon_{n}^{2}}{2 \mu_{n} b_{n}}-\frac{\kappa_{n} g_{n}\left(\bar{x}_{n}\right) z_{n} \beta_{n}\left(z_{n}\right)}{k_{b n}^{2}-z_{n}^{2}} \\
& \left.+\frac{l_{n}}{2}+\frac{1}{4}+\frac{1}{2} \sum_{j=1}^{n} \rho_{n j}^{2}\left(x_{j}\left(t-T_{j}(t)\right)\right)\right), \\
& +\frac{\sigma_{n 1}}{2 b_{n}} \Theta_{n}^{2}-\frac{\sigma_{n 1}}{2 b_{n}} \widetilde{\omega}_{n}^{2}-\frac{\sigma_{n 2} \zeta_{1}}{b_{n}^{h}} \widetilde{\omega}_{n}^{1+h}+\frac{\sigma_{n 2} \zeta_{2}}{b_{n}^{h}} \varpi_{n}^{1+h} \\
& -\frac{g_{n-1}\left(\bar{x}_{n-1}\right) z_{n-1} z_{n}}{k_{b(n-1)}^{2}-z_{n-1}^{2}},
\end{aligned}
$$

where $\zeta_{1}$ and $\zeta_{2}$ are defined in Lemma 4 .

To handle the time delay, define the Lyapunov-Krasovskii functional as follows:

$$
V_{U_{n}}=\frac{e^{-\gamma\left(t-T_{\max }\right)}}{2\left(1-\bar{T}_{\max }\right)} \sum_{j=1}^{n} \int_{t-T_{j}(t)}^{t} e^{\gamma s} \rho_{n j}^{2}\left(x_{j}(s)\right) \mathrm{d} s,
$$


where $\gamma>0$ is a positive constant. By using Assumption 5, we obtain that the derivative of $V_{U n}$ is

$$
\begin{aligned}
\dot{V}_{U_{n}}= & \frac{e^{-\gamma\left(t-T_{\max }\right)}}{2\left(1-\bar{T}_{\max }\right)} \sum_{j=1}^{n}\left[e^{\gamma t} \rho_{n j}^{2}\left(x_{j}(t)\right)-e^{\gamma\left(t-T_{j}(t)\right)} \rho_{n j}^{2}\right. \\
& \left.\cdot\left(x_{j}\left(t-T_{j}(t)\right)\right)\left(1-\dot{T}_{j}(t)\right)\right]-\gamma V_{U_{n}}, \\
\leq & \sum_{j=1}^{n} \frac{e^{\gamma T_{\max }}}{2\left(1-\bar{T}_{\max }\right)} \rho_{n j}^{2}\left(x_{j}(t)\right)-\frac{1}{2} \sum_{j=1}^{n} \rho_{n j}^{2}\left(x_{j}\left(t-T_{j}(t)\right)\right)-\gamma V_{U_{n}} .
\end{aligned}
$$

From equations (60) and (62), we have

$$
\begin{aligned}
\dot{V}_{z_{n}}+\dot{V}_{U_{n}} \leq & -\frac{b_{n} c_{n} z_{n}^{2}}{k_{b n}^{2}-z_{n}^{2}}+\frac{\varepsilon_{n}^{2}}{2 \mu_{n} b_{n}}-\frac{\kappa_{n} g_{n}\left(\bar{x}_{n}\right) z_{n} \beta_{n}\left(z_{n}\right)}{k_{b n}^{2}-z_{n}^{2}} \\
& +\frac{l_{n}}{2}+\frac{1}{4}+\frac{\sigma_{n 1}}{2 b_{n}} \varpi_{n}^{2}-\frac{\sigma_{n 1}}{2 b_{n}} \widetilde{\omega}_{n}^{2}, \\
& -\frac{\sigma_{n 2} \zeta_{1}}{b_{n}^{h}} \widetilde{\omega}_{n}^{1+h}+\frac{\sigma_{n 2} \zeta_{2}}{b_{n}^{h}} \Theta_{n}^{1+h}-\frac{g_{n-1}\left(\bar{x}_{n-1}\right) z_{n-1} z_{n}}{k_{b(n-1)}^{2}-z_{n-1}^{2}} \\
& +\Phi_{n}-\gamma V_{U_{n}},
\end{aligned}
$$

where $\Phi_{n}=\sum_{j=1}^{n}\left(e^{\gamma T_{\max }} /\left(2\left(1-\bar{T}_{\max }\right)\right)\right) \rho_{n j}^{2}\left(x_{j}(t)\right)$.

3.2. Stability Analysis. In this subsection, we present the stability analysis of the resulting closed-loop system. The main results are presented by the following theorem.

Theorem 1 Consider the nonlinear system (1) with Assumptions 1-5. Let the actual control input and the NN adaptive law be designed as (49) and (50), respectively. If the initial conditions satisfy $V(0) \leq \Delta,\left|z_{i}(0)\right| \leq k_{b i}$, in which $\Delta>k_{b i}$ is any positive constant for $i=1,2, \ldots, n$ and $k_{b i}$ are properly chosen, such that $k_{c 1}>k_{b 1}+A_{0}$ and $k_{c i}>\bar{w}_{i}+k_{b i}$ with $\bar{w}_{i}=\sup \left\{w_{i}\right\}$ for $i=2,3, \ldots, n$, one has that all internal signals $z_{i}, \widetilde{\mathrm{\omega}}_{i}$ and $y_{i+1}$ in the closed-loop system are semiglobally uniformly ultimately bounded and the tracking error will converge into the arbitrarily small regions in a finite time. Meanwhile, each state $x_{i}$ will remain in the set $\Omega_{x_{i}}$; that is, the full-state constraints are never violated.

Proof. Construct the overall Lyapunov function candidate

$$
V=\sum_{i=1}^{n} V_{z_{i}}+\sum_{i=1}^{n} V_{U_{i}}+\sum_{i=1}^{n-1} V_{y_{i}}
$$

where $V_{y_{i}}=\left(a_{i}^{2} / 2 b_{i}\right) y_{i+1}^{2}$ and $V_{z_{i}}, V_{U_{i}}$ are defined in (35) and (44), respectively.

From (28), (29), and (47), the derivative of $\sum_{i=1}^{n-1} V_{y_{i}}$ is

$$
\begin{aligned}
\sum_{i=1}^{n-1} \dot{V}_{y_{i}} \leq & -\sum_{i=1}^{n-1} \frac{a_{i}^{2}}{b_{i}}\left(\tau_{(i+1) 1} y_{i+1}^{2}+\tau_{(i+1) 2} y_{i+1}^{1+h}\right) \\
& +\sum_{i=1}^{n-1} \frac{a_{i}^{2}}{b_{i}} y_{i+1} \lambda_{i+1}\left(\bar{z}_{i+1}, \overline{\widehat{\widehat{\omega}}}_{i}, y_{2}, y_{3}, \ldots, y_{i+1}, \dot{y}_{r}, \ddot{y}_{r}\right) .
\end{aligned}
$$

Define a compact set as $\Omega_{n}=\left\{\left(\bar{z}_{n}, \overline{\widehat{\widehat{\omega}}}_{n}, y_{2}, y_{3}, \ldots, y_{n}\right): V \leq \Delta\right\}$ with $\Delta$ being a positive constant. If $V \leq \Delta$, together with Assumption 4 and (65), it can be obtained that there exists a positive constant $\Lambda_{i+1}(i=1,2, \ldots, n-1)$, such that $\lambda_{i+1}(\cdot) \leq \Lambda_{i+1}$ on the compact set $\Omega_{n} \times \Omega_{d}$. Then, applying Young's inequality to (65) yields

$$
\begin{aligned}
\sum_{i=1}^{n-1} \dot{V}_{y_{i}} \leq & -\sum_{i=1}^{n-1} \frac{a_{i}^{2}}{b_{i}}\left(\tau_{(i+1) 1}-\frac{1}{2 \chi_{i+1}}\right) y_{i+1}^{2}-\sum_{i=1}^{n-1} \frac{a_{i}^{2}}{b_{i}} \tau_{(i+1) 2} y_{i+1}^{1+h} \\
& +\sum_{i=1}^{n-1} \frac{a_{i}^{2}}{2 b_{i}} \Lambda_{i+1}^{2} \chi_{i+1}
\end{aligned}
$$

where $\chi_{i+1}$ are positive constants.

According to the above analysis, we can obtain the derivative of the overall Lyapunov function candidate $V$ as

$$
\begin{aligned}
\dot{V} \leq & -\sum_{i=1}^{n} \frac{b_{i} c_{i} z_{i}^{2}}{k_{b i}^{2}-z_{i}^{2}}-\sum_{i=1}^{n} \frac{\kappa_{i} g_{i}\left(\bar{x}_{i}\right) z_{i} \beta_{i}\left(z_{i}\right)}{k_{b i}^{2}-z_{i}^{2}}-\sum_{i=1}^{n} \frac{\sigma_{i 1}}{2 b_{i}} \widetilde{\omega}_{i}^{2} \\
& -\sum_{i=1}^{n} \frac{\sigma_{i 2} \zeta_{1}}{b_{i}^{h}} \widetilde{\omega}_{i}^{1+h}-\sum_{i=1}^{n-1} \hat{\tau}_{(i+1) 1} y_{i+1}^{2}, \\
& -\sum_{i=1}^{n-1} \frac{a_{i}^{2}}{b_{i}} \tau_{(i+1) 2} y_{i+1}^{1+h}-\gamma \sum_{i=1}^{n} V_{U_{i}}+d_{0},
\end{aligned}
$$

where

$$
\begin{aligned}
\widehat{\tau}_{(i+1) 1}= & \frac{a_{i}^{2}}{b_{i}}\left(\tau_{(i+1) 1}-\frac{1}{2 \mu_{i}}-\frac{1}{2 \chi_{i+1}}\right), \\
d_{0}= & \sum_{i=1}^{n}\left(\frac{\varepsilon_{i}^{2}}{2 \mu_{i} b_{i}}+\frac{l_{i}}{2}+\frac{1}{4}+\frac{\sigma_{i 1}}{2 b_{i}}{\omega_{i}^{2}}^{2}+\frac{\sigma_{i 2} \zeta_{2}}{b_{i}^{h}}{\omega_{i}^{1+h}}^{1+h} \Phi_{i}\right) \\
& +\sum_{i=1}^{n-1}\left(\frac{a_{i}^{2}}{2 b_{i}} \Lambda_{i+1}^{2} \chi_{i+1}\right) .
\end{aligned}
$$

Here, we choose $\tau_{(i+1) 1}>\left(1 / 2 \mu_{i}\right)+\left(1 / 2 \chi_{i+1}\right)$, such that $\widehat{\tau}_{(i+1) 1}>0$.

From the definition of $\beta_{i}\left(z_{i}\right)(i=1,2, \ldots, n)$ in (15), (34), and (51), the following two cases should be considered.

Case 1: When $\left|z_{i}\right|<\tau_{i}, i=1,2, \ldots, n$, substituting $\beta_{i}\left(z_{i}\right)=\iota_{i 1} z_{i}+l_{i 2} z_{i}^{3}$ into (67) gives 


$$
\begin{aligned}
\dot{V} \leq & -\sum_{i=1}^{n}\left(c_{i}+\kappa_{i} \iota_{i 1}\right) \frac{b_{i} z_{i}^{2}}{k_{b i}^{2}-z_{i}^{2}}-\sum_{i=1}^{n} \frac{\kappa_{i} b_{i} \iota_{i 2} z_{i}^{4}}{k_{b i}^{2}-z_{i}^{2}}-\sum_{i=1}^{n} \frac{\sigma_{i 1}}{2 b_{i}} \widetilde{\mathrm{\omega}}_{i}^{2} \\
& -\sum_{i=1}^{n} \frac{\sigma_{i 2} \zeta_{1}}{b_{i}^{h}} \widetilde{\mathrm{\omega}}_{i}^{1+h}, \\
& -\sum_{i=1}^{n-1} \widehat{\tau}_{(i+1) 1} y_{i+1}^{2}-\sum_{i=1}^{n-1} \frac{a_{i}^{2}}{b_{i}} \tau_{(i+1) 2} y_{i+1}^{1+h}-\gamma \sum_{i=1}^{n} V_{U i}+d_{0}, \\
\leq & -\sum_{i=1}^{n}\left(c_{i}+\kappa_{i} \iota_{i 1}\right) \frac{b_{i} z_{i}^{2}}{k_{b i}^{2}-z_{i}^{2}}-\sum_{i=1}^{n} \frac{\sigma_{i 1} b_{i}}{\widetilde{\Phi}_{i}^{2}} \\
& -\sum_{i=1}^{n-1} \widehat{\tau}_{(i+1) 1} y_{i+1}^{2}-\gamma \sum_{i=1}^{n} V_{U_{i}}+d_{0} .
\end{aligned}
$$

Noting (69), we can have

$$
\dot{V} \leq-v V+d_{0}
$$

with $v=\min \left\{2 b_{1}\left(c_{1}+\kappa_{1} \iota_{11}\right), \ldots, 2 b_{n}\left(c_{n}+\kappa_{n} \iota_{n 1}\right), \rho_{1} \sigma_{11}\right.$, $\left.\ldots, \rho_{n} \sigma_{n 1}, \gamma,\left(2 b_{1} \widehat{\tau}_{(2,1)} / a_{1}^{2}\right), \ldots,\left(2 b_{n-1} \widehat{\tau}_{(n, 1)} / a_{n-1}^{2}\right)\right\}$, which further implies that all the internal signals are uniformly ultimately bounded.

Case 2: When $\left\|z_{i}\right\| \geq \tau_{i}, i=1,2, \ldots, n$, substituting $\beta_{i}\left(z_{i}\right)=z_{i}^{h}\left(k_{b i}^{2}-z_{i}^{2}\right)^{(1-h / 2)}$ into (67) gives

$$
\begin{aligned}
& \dot{V} \leq-\sum_{i=1}^{n} \frac{b_{i} c_{i} z_{i}^{2}}{k_{b i}^{2}-z_{i}^{2}}-\sum_{i=1}^{n} \frac{\kappa_{i} g_{i}\left(\bar{x}_{i}\right) z_{i}^{1+h}}{\left(k_{b i}^{2}-z_{i}^{2}\right)^{(1+h / 2)}}-\sum_{i=1}^{n} \frac{\sigma_{i 1}}{2 b_{i}} \widetilde{\varpi}_{i}^{2}-\sum_{i=1}^{n} \frac{\sigma_{i 2} \zeta_{1}}{b_{i}^{h}} \widetilde{\omega}_{i}^{1+h}-\sum_{i=1}^{n-1} \widehat{\tau}_{(i+1) 1} y_{i+1}^{2}, \\
& -\sum_{i=1}^{n-1} \frac{a_{i}^{2}}{b_{i}} \tau_{(i+1) 2} y_{i+1}^{1+h}-\frac{\gamma}{2} \sum_{i=1}^{n} V_{U i}-\frac{\gamma}{2}\left[\sum_{i=1}^{n} V_{U_{i}}\right]^{(1+h / 2)}+\frac{\gamma}{2} \frac{1-h}{2}\left(\frac{1+h}{2}\right)^{(1+h / 1-h)}+d_{0} \\
& \leq-\sum_{i=1}^{n} \frac{b_{i} c_{i} z_{i}^{2}}{k_{b i}^{2}-z_{i}^{2}}-\sum_{i=1}^{n} \frac{\kappa_{i} g_{i}\left(\bar{x}_{i}\right) z_{i}^{1+h}}{\left(k_{b i}^{2}-z_{i}^{2}\right)^{(1+h / 2)}}-\sum_{i=1}^{n} \frac{\sigma_{i 1}}{2 b_{i}} \widetilde{\omega}_{i}^{2}-\sum_{i=1}^{n} \frac{\sigma_{i 2} \zeta_{1}}{b_{i}^{h}} \widetilde{\omega}_{i}^{1+h}-\sum_{i=1}^{n-1} \widehat{\tau}_{(i+1) 1} y_{i+1}^{2}, \\
& -\sum_{i=1}^{n-1} \frac{a_{i}^{2}}{b_{i}} \tau_{(i+1) 2} y_{i+1}^{1+h}-\frac{\gamma}{2} \sum_{i=1}^{n} V_{U i}-\frac{\gamma}{2 n^{(1-h / 2)}}\left[\sum_{i=1}^{n} V_{U_{i}}\right]^{(1+h / 2)}+\frac{\gamma}{2} \frac{1-h}{2}\left(\frac{1+h}{2}\right)^{(1+h / 1-h)}+d_{0}, \\
& \leq-v_{1} V-v_{2} V^{(1+h / 2)}+d_{1} \text {, }
\end{aligned}
$$

where

$$
\left.\begin{array}{l}
v_{1}=\min \left\{2 b_{1} c_{1}, \ldots, 2 b_{n} c_{n}, \sigma_{11} \rho_{1}, \ldots, \sigma_{n 1} \rho_{n}, \frac{\gamma}{2}, \frac{2 b_{1} \widehat{\tau}_{21}}{a_{1}^{2}}, \ldots, \frac{2 b_{n-1} \widehat{\tau}_{n 1}}{a_{n-1}^{2}}\right\}, \\
v_{2}=\min \left\{\begin{array}{l}
b_{1} c_{1} 2^{(1+h / 2)}, \ldots, b_{n} c_{n} 2^{(1+h / 2)}, \frac{\sigma_{12} \zeta_{1}}{b_{1}^{h}}\left(\frac{2 b_{1} \rho_{1}}{\sigma_{11}}\right)^{(1+h / 2)}, \ldots, \frac{\sigma_{n 2} \zeta_{1}}{b_{n}^{h}}\left(\frac{2 b_{n} \rho_{n}}{\sigma_{n 1}}\right)^{(1+h / 2)}, \\
\frac{\gamma}{2 n^{(1-h / 2)}}, \frac{a_{1}^{2} \tau_{22}}{b_{1}}\left(\frac{2 b_{1}}{a_{1}^{2}}\right)^{(1+h / 2)}, \ldots, \frac{a_{n-1}^{2} \tau_{n 2}}{b_{n-1}}\left(\frac{2 b_{n-1}}{a_{n-1}^{2}}\right)^{(1+h / 2)}
\end{array}\right. \\
d_{1}=d_{0}+\frac{\gamma}{2} \frac{1-h}{2}\left(\frac{1+h}{2}\right)^{(1+h / 1-h)} .
\end{array}\right\},
$$


By virtue of [[59], Th.5.2], there always exists a finitetime $t^{*}$, such that $V \geq\left(2 d_{1} / v_{2}\right)^{(1+h / 2)}$ for all $t \in\left[0, t^{*}\right]$. Thus, for all $t \in\left[0, t^{*}\right]$, one has $\dot{V} \leq-v_{1} V-\left(v_{2} / 2\right) V^{(1+h / 2)}$, and it then comes from Lemma 3 that the fast finite-time stability of the closed-loop system can be ensured with a finite settling time $\quad T^{*} \leq\left(2 /\left(v_{1}(1-h)\right)\right) \log \left(\left(2 v_{1} V^{(1-h / 2)}(0)+v_{2}\right) / v_{2}\right)$. Furthermore, it is readily seen that $t^{*} \leq T^{*}$. Therefore, $\forall t>T^{*}, V \leq\left(2 d_{1} / v_{2}\right)^{(2 / 1+h)}$. Then, the internal error signals $z_{i}, \widetilde{\varpi}_{i}$, and $y_{i+1}$ will converge into the following compact sets:

$$
\begin{aligned}
\left|z_{i}\right| & \leq k_{b i}\left(1-e^{-2\left(2 d_{1} / v_{2}\right)^{(2 / 1+h)}}\right)^{(1 / 2)}, \quad i=1, \ldots, n, \\
\left|\widetilde{\varpi}_{i}\right| & \leq\left(2 \rho_{i} b_{i}\right)^{(1 / 2)}\left(\frac{2 d_{1}}{v_{2}}\right)^{(1 / 1+h)}, \quad i=1, \ldots, n, \\
\left|y_{i+1}\right| & \leq\left(\frac{2 b_{i}}{a_{i}^{2}}\right)^{(1 / 2)}\left(\frac{2 d_{1}}{v_{2}}\right)^{(1 / 1+h)}, \quad i=1, \ldots, n-1,
\end{aligned}
$$

in a finite-time $T^{*}$ with $T^{*} \leq\left(2 /\left(v_{1}(1-h)\right)\right) \log \left(\left(2 v_{1}\right.\right.$ $\left.\left.V^{(1-h / 2)}(0)+v_{2}\right) / v_{2}\right)$. It is readily seen that the regions (73) can be made as small as possible by adjusting $\left(2 d / v_{2}\right)$ with proper control parameters.

Then, we will prove that the full-state constraints are never violated. According to [[60], Lemma 1], we can conclude from (70) and (71) that $\left|z_{i}\right| \leq k_{b i}, i=1, \ldots, n$, for all $t \geq 0$. Noting that $\left|y_{r}\right| \leq A_{0}$ from Assumption 4 and $z_{1}=x_{1}-y_{r}$, we have that $\left|x_{1}\right| \leq k_{b 1}+A_{0}$. To get $x_{2} \leq k_{c 2}$, we need to show the boundedness of $w_{2}$. From (73), one has that $y_{2}$ is bounded and $b_{1} \widehat{\Phi}_{1}=\Phi_{1}-\widetilde{\Phi}_{1}$ is also bounded. With the proper choices of $\iota_{1}$ and $l_{2}, \alpha_{1}$ is a continuous function of $\widehat{\mathrm{\omega}}_{1}, x_{1}$, and $\dot{y}_{r}$. Then, there exists an upper bound $\bar{w}_{2}$, such that $w_{2}=\left|y_{2}+\alpha_{1}\right| \leq \bar{w}_{2}$. From $z_{2}=x_{2}-w_{2}$ and $z_{2}<k_{b 2}$, we get that $\left|x_{2}\right| \leq\left|z_{2}\right|+\left|w_{2}\right| \leq k_{c 2}$. Similarly and iteratively, we have that $\alpha_{i-1}$ and $y_{i}$ for $i=3, \ldots, n$ are bounded, which together with $z_{i}<k_{b i}$ ensures that $\left|x_{i}\right| \leq k_{c i}, i=3, \ldots, n$. Therefore, each state $x_{i}, i=3, \ldots, n$ will remain in the set $\Omega_{x_{i}}$. The proof is completed.

\section{Simulation Results}

Example 1. Consider the following nonlinear system:

$$
\left\{\begin{array}{l}
\dot{\xi}=-\xi+0.5 x_{1}^{2} \sin \left(x_{1} t\right), \\
\dot{x}_{1}=x_{2} e^{-0.5 x_{1}}+\left(1+x_{1}^{2}\right) x_{2}+\delta_{1}\left(\xi, x_{1}, x_{2}, t\right)+2 x_{1}^{2}\left(t-T_{1}(t)\right), \\
\dot{x}_{2}=x_{1} x_{2}^{2}+2.5 u(t)+\delta_{2}\left(\xi, x_{1}, x_{2}, t\right)+0.2 x_{2}^{2}\left(t-T_{2}(t)\right), \\
y=x_{1},
\end{array}\right.
$$

where $\delta_{1}\left(\xi, x_{1}, x_{2}, t\right)=0.2 \xi x_{1} \sin \left(x_{2} t\right), \delta_{2}\left(\xi, x_{1}, x_{2}, t\right)=0.1 \xi$ $\cos \left(0.5 x_{2} t\right), T_{1}(t)=0.2(4+\sin t), T_{2}=4+0.5 \sin t$, and the dynamic signal $\dot{r}=-r+2.5 x_{1}^{4}+0.625$. The desired tracking trajectory $y_{r}=\sin (0.5 t) . u$ is the control input. The design parameters of the controller are taken as $k_{b 1}=0.4, k_{b 2}=2, c_{1}=$ $10, c_{2}=15, \tau_{1}=\tau_{2}=0.01, \sigma_{11}=\sigma_{12}=0.01, \rho_{1}=\rho_{2}=50, l_{1}=l_{2}=$ $1, T=0.001, \mu_{1}=\mu_{2}=3, \kappa_{1}=\kappa_{2}=0.5, h=0.6$. There are 68

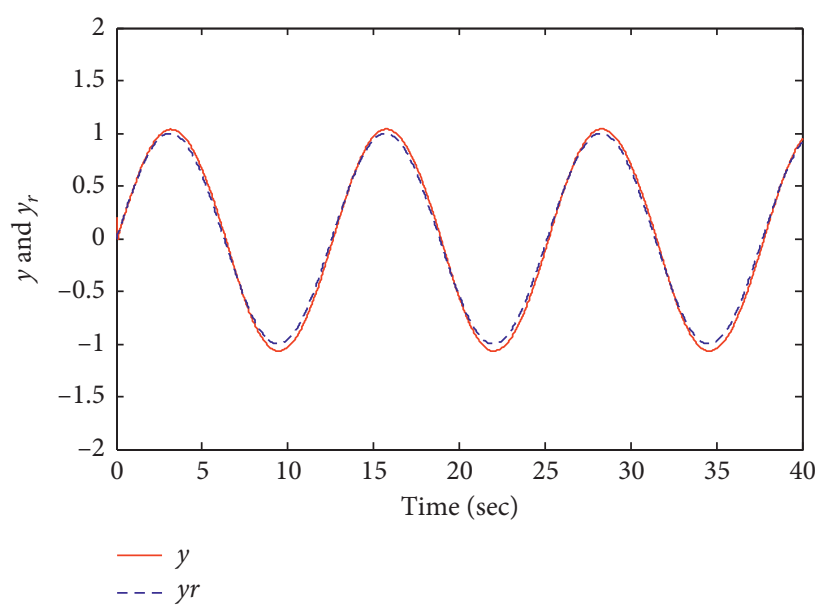

Figure 1: Output $y$ and desired trajectory $y_{r}$.

nodes with the center placed on $[-2,2] \times[-2,2] \times[-2,2] \times$ $[-2,2]$ and the width of Gaussian functions is $\eta_{1}=1$ in the first $\mathrm{RBF}$ vector. There are 85 nodes with the center placed on $[-2,2] \times[-2,2] \times[-2,2] \times[-2,2] \times[-2,2]$ and the width of Gaussian functions is $\eta_{2}=1$ in the second $\mathrm{RBF}$ vector. With the initial conditions, $x_{1}(0)=0.2, x_{2}(0)=0.1, w_{1}(0)=0.1$, $\widehat{\Phi}_{1}=2, \widehat{\Phi}_{2}=0.5, r(0)=0.1$. Simulation results are shown in Figures 1-6. The profiles of the system output $y$ and the desired signal $y_{r}$ are shown in Figure 1, which indicates that the output $y$ follows the specified desire trajectory $y_{r}$. From Figure 2, we know that all state constraints are not violated.

Example 2. A Spring-Mass-Damper system is provided in this part. The system model is as follows:

$$
\left\{\begin{array}{l}
\dot{p}=V \\
\mathrm{MV}=-\mathrm{KP}-\mathrm{CV}+F
\end{array}\right.
$$

where $P, V$ and $F$ are the position, the velocity, and the force applied to the object, respectively. Let $x_{1}=P, x_{2}=V, u=F$. Assuming that the controlled system (75) gives unmodeled dynamics and time delay, let $\delta_{1}\left(\xi, x_{1}, x_{2}, t\right)=0.2 \xi x_{1}$ $\sin \left(x_{2} t\right), \delta_{2}\left(\xi, x_{1}, x_{2}, t\right)=0.1 \xi \cos \left(0.5 x_{2} t\right), T_{1}(t)=0.2(4+$ $\sin t), T_{2}=4+0.5 \sin t$, and the dynamic signal $\dot{r}=-r+$ $2.5 x_{1}^{4}+0.625$. Then, system (75) can be rewritten as

$\left\{\begin{array}{l}\dot{\xi}=-\xi+0.5 x_{1}^{2} \sin \left(x_{1} t\right), \\ \dot{x}_{1}=x_{2}+\delta_{1}\left(\xi, x_{1}, x_{2}, t\right)+2 x_{1}^{2}\left(t-T_{1}(t)\right), \\ \dot{x}_{2}=-\frac{K}{M} x_{1}-\frac{C}{M} x_{2}+\frac{1}{M} u(t)+\delta_{2}\left(\xi, x_{1}, x_{2}, t\right)+0.2 x_{2}^{2}\left(t-T_{2}(t)\right), \\ y=x_{1},\end{array}\right.$

The desired tracking trajectory $y_{r}=\sin (0.5 t)+0.5 \sin (t)$. The design parameters of the controller are taken as $k_{b 1}=$ $0.4, k_{b 2}=2, c_{1}=10, c_{2}=15, \tau_{1}=\tau_{2}=0.01, \sigma_{11}=\sigma_{12}=0.01, \rho_{1}=$ $\rho_{2}=50, l_{1}=l_{2}=1, T=0.001, \mu_{1}=\mu_{2}=3, \kappa_{1}=\kappa_{2}=0.5, \quad h=0.6$. 


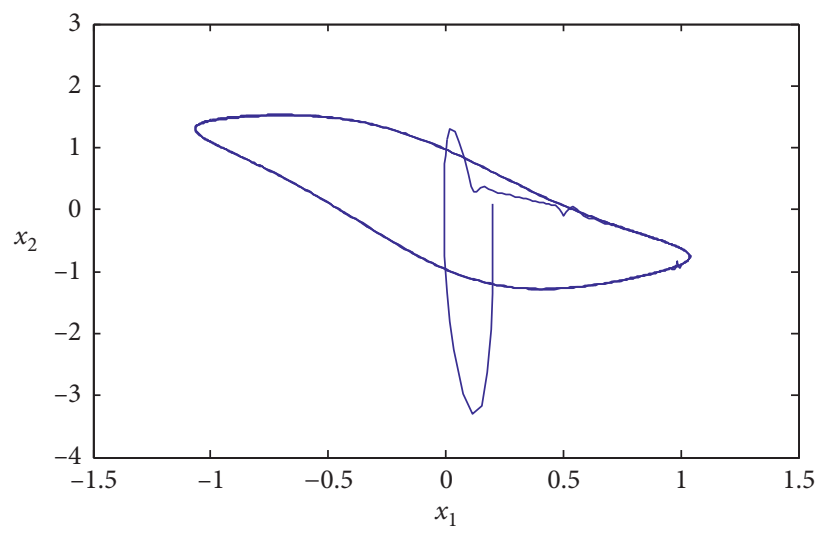

Figure 2: Phase portrait of states $x_{1}$ and $x_{2}$.

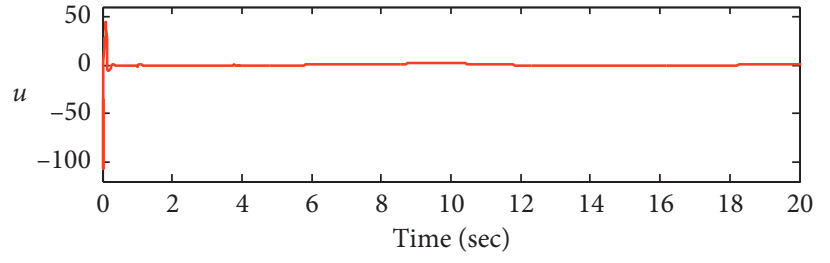

(a)

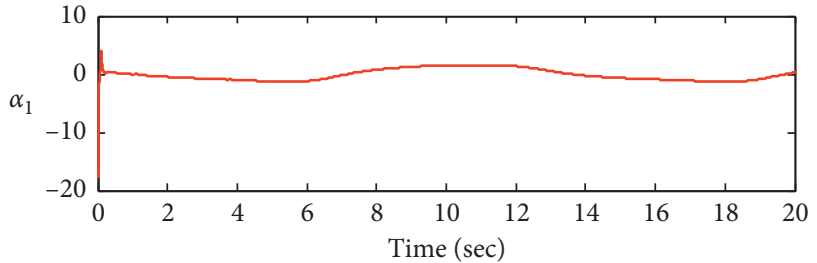

(b)

Figure 3: Profiles of control inputs $u$ and $\alpha_{1}$.

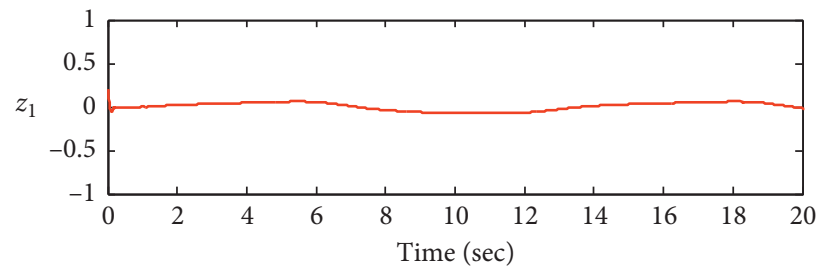

(a)

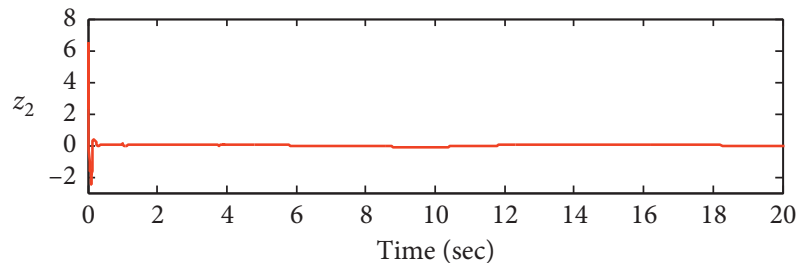

(b)

Figure 4: Profiles of the tracking errors $z_{1}$ and $z_{2}$.

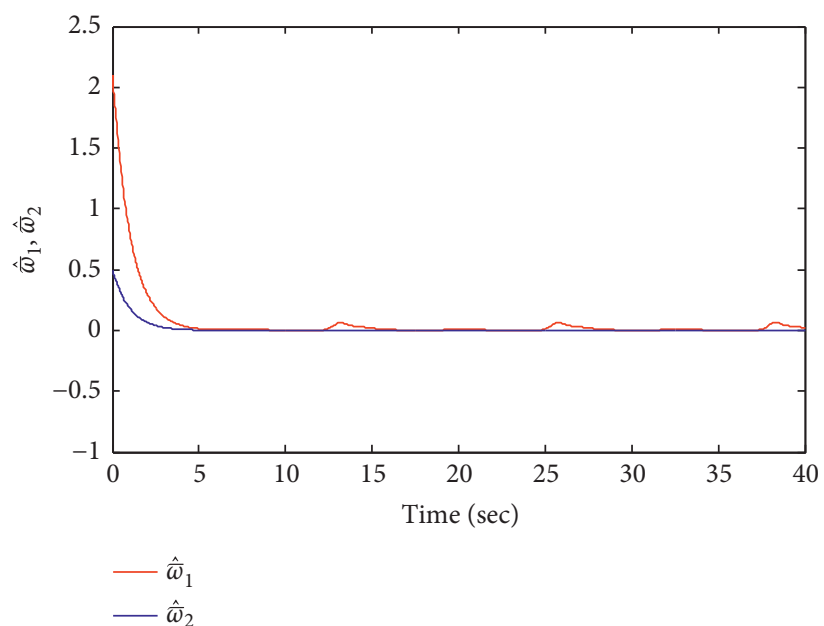

FIGURE 5: Estimated parameters $\widehat{\omega}_{1}$ and $\widehat{\omega}_{2}$. 


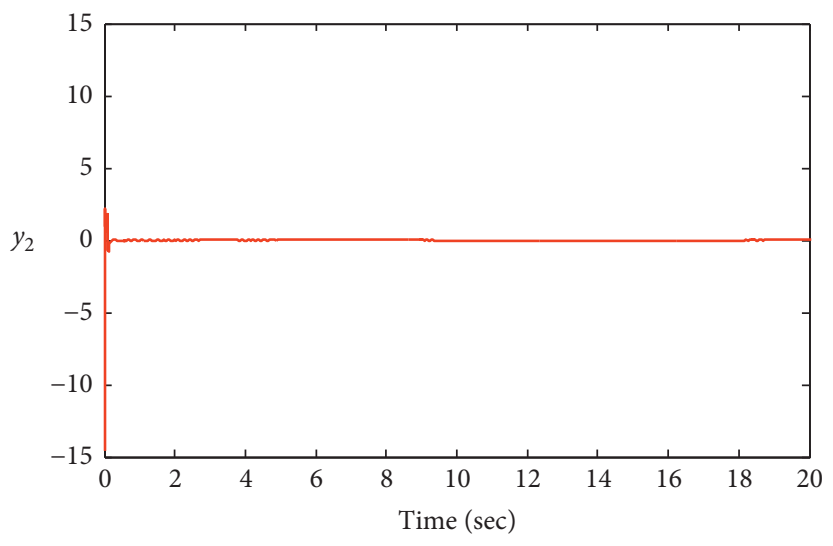

Figure 6: Profiles of the boundary layer error $y_{2}$.

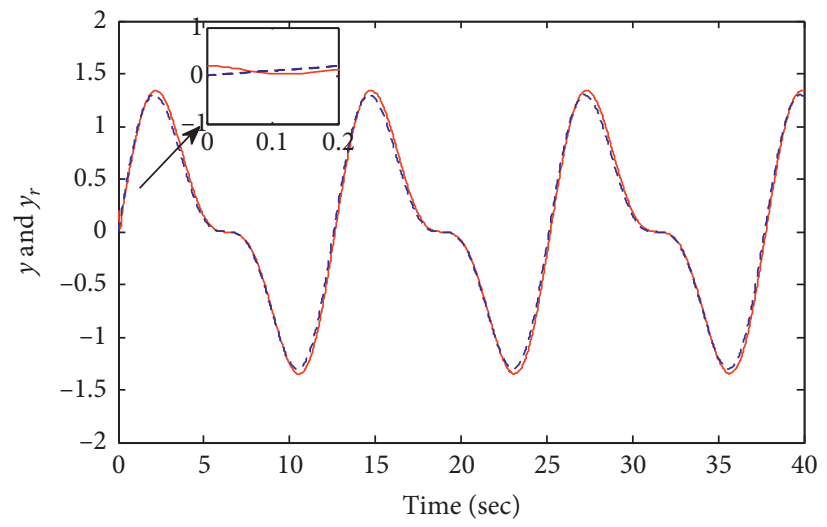

$-\mathrm{y}$

FIgURE 7: Output $y$ and desired trajectory $y_{r}$.

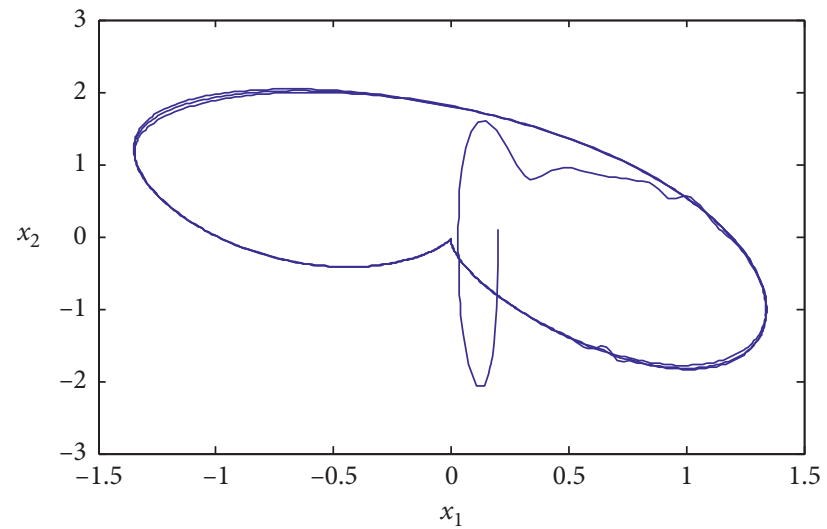

Figure 8: Phase portrait of states $x_{1}$ and $x_{2}$.

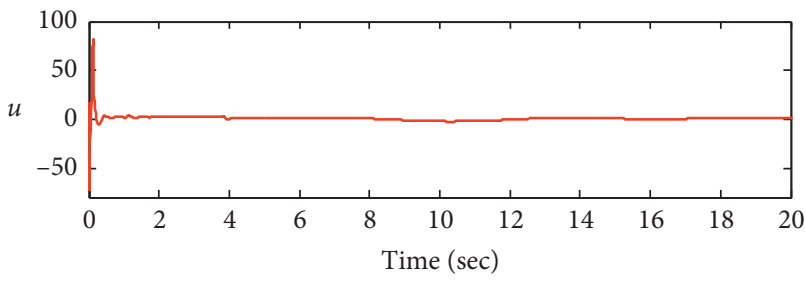

(a)

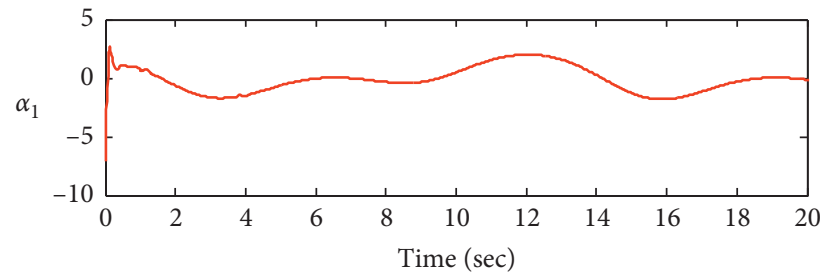

(b)

Figure 9: Profiles of control inputs $u$ and $\alpha_{1}$. 

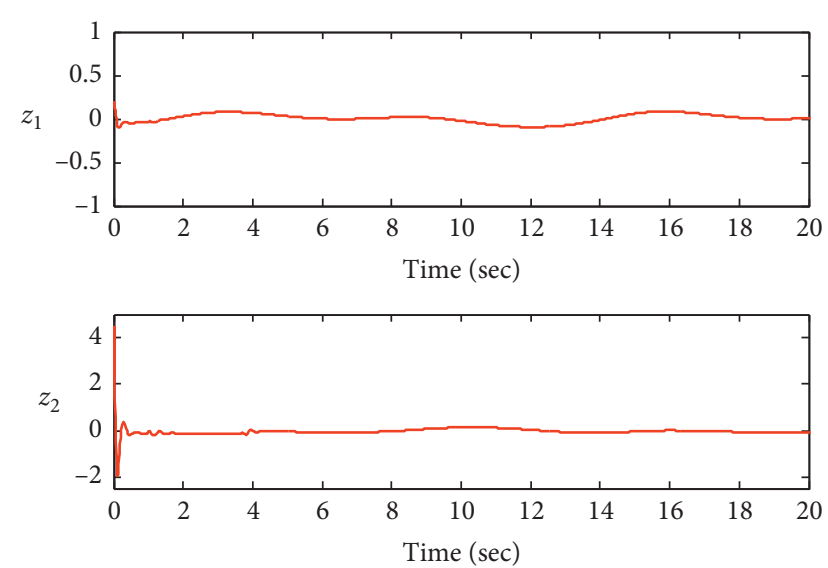

Figure 10: Profiles of the tracking errors $z_{1}$ and $z_{2}$.

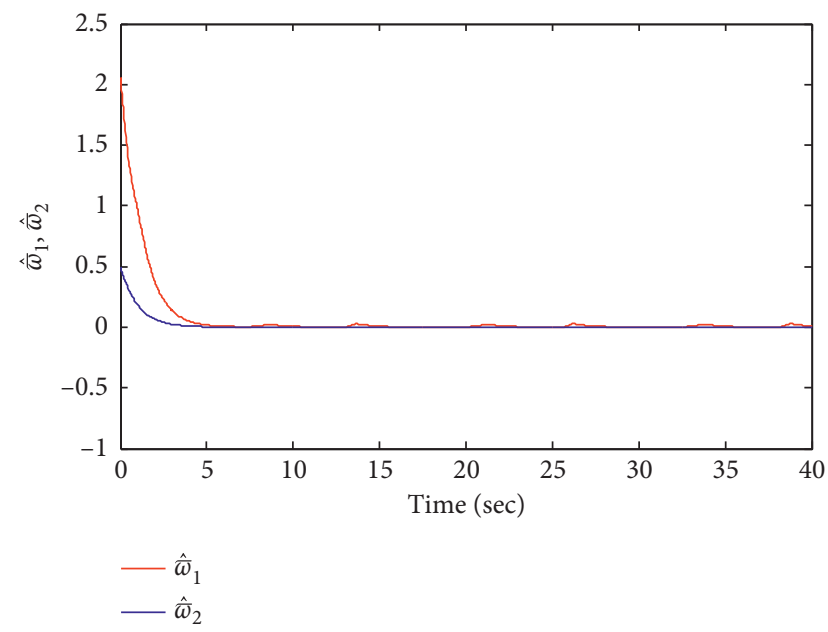

FIGURE 11: Estimated parameters $\widehat{\widehat{\omega}}_{1}$ and $\widehat{\omega}_{2}$.

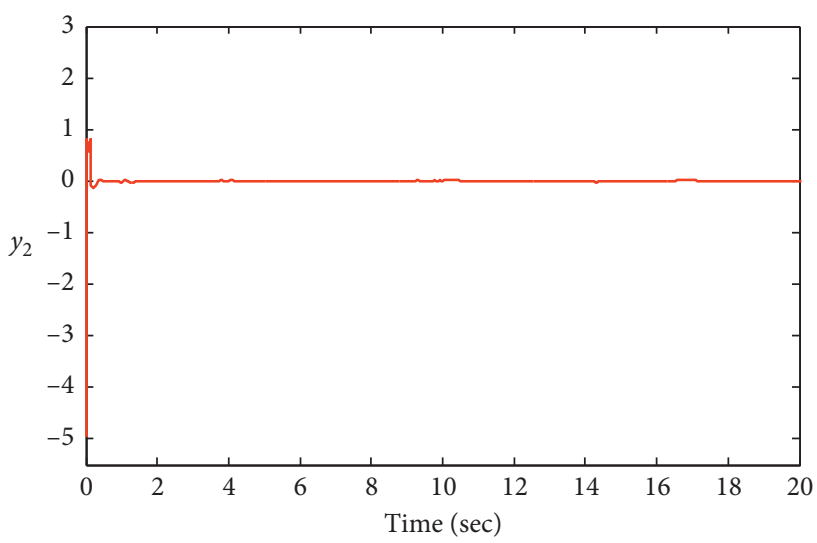

Figure 12: Profiles of the boundary layer error $y_{2}$.

There are 68 nodes with the center placed on $[-2,2] \times[-2,2] \times$ $[-2,2] \times[-2,2]$ and the width of Gaussian functions is $\eta_{1}=1$ in the first RBF vector. There are 85 nodes with the center placed on $[-2,2] \times[-2,2] \times[-2,2] \times[-2,2] \times[-2,2]$ and the width of Gaussian functions is $\eta_{2}=1$ in the second RBF vector.
With the initial conditions, $x_{1}(0)=0.2, x_{2}(0)=0.1, w_{1}(0)=$ $0.1, \widehat{\oplus}_{1}=2, \widehat{\omega}_{2}=0.5, r(0)=0.1$. Simulation results are shown in Figures 7-12.

\section{Conclusions}

The problem of finite-time tracking control for a class of uncertain nonstrict-feedback state-delayed nonlinear systems with full-state constraints and unmodeled dynamics has been proposed in this paper. Unmodeled dynamics is dealt with by introducing a dynamical signal and the uncertain terms produced by time-varying state delays are compensated for by constructing appropriate Lyapunov-Krasovskii functionals. By utilizing a smooth switch between the fractional and cubic form state feedback, novel $C^{1}$ smooth finite-time NN control laws have been provided for nonlinear systems with full-state constraints. Based on a modified DSC method and adaptive NN control, together with the BLFs, the fast finite-time control performance of the closed-loop nonlinear systems can be ensured, while the full-state constraints are never violated. Theoretical proofs and experimental simulation show that all the internal signals in the closed-loop system are uniformly bounded, and the tracking error signals can converge into compact sets in a finite time with sufficient accuracy, respectively. To extend this control scheme to solve the finite-time tracking control problem for some more complicated systems, such as MIMO nonlinear systems, switched nonlinear systems are also the direction of our future efforts.

\section{Data Availability}

This paper is a theoretical study and no data were used to support this study.

\section{Conflicts of Interest}

The authors declare that they do not have any financial or nonfinancial conflicts of interest.

\section{Acknowledgments}

This work was supported by the National Natural Science Foundation of China (61603003 and 61472466).

\section{References}

[1] S. S. Ge and C. Wang, "Adaptive NN control of uncertain nonlinear pure-feedback systems," Automatica, vol. 38, no. 4, pp. 671-682, 2002.

[2] J. Wu, Z.-G. Wu, J. Li, G. Wang, H. Zhao, and W. Chen, "Practical adaptive fuzzy control of nonlinear pure-feedback systems with quantized nonlinearity input," IEEE Transactions on Systems, Man, and Cybernetics: Systems, vol. 49, no. 3, pp. 638-648, 2019.

[3] H. Wang, B. Chen, X. Liu, K. Liu, and C. Lin, "Robust adaptive fuzzy tracking control for pure-feedback stochastic nonlinear systems with input constraints," IEEE Transactions on Cybernetics, vol. 43, no. 6, pp. 2093-2104, 2013. 
[4] Y. Li, S. Tong, and T. Li, "Adaptive fuzzy output feedback dynamic surface control of interconnected nonlinear purefeedback systems," IEEE Transactions on Cybernetics, vol. 45, no. 1, pp. 138-149, 2015.

[5] J. Na, X. Ren, and D. Zheng, "Adaptive control for nonlinear pure-feedback systems with high-order sliding mode observer," IEEE Transactions on Neural Networks and Learning Systems, vol. 24, no. 3, pp. 370-382, 2013.

[6] B. Chen, X. Liu, K. Liu, and C. Lin, "Fuzzy-approximationbased adaptive control of strict-feedback nonlinear systems with time delays," IEEE Transactions on Fuzzy Systems, vol. 18, no. 5, pp. 883-892, 2010.

[7] W. Chen, L. Jiao, J. Li, and R. Li, “Adaptive NN backstepping output-feedback control for stochastic nonlinear strict-feedback systems with time-varying delays," IEEE Transactions on Systems, Man, and Cybernetics. Part B, Cybernetics: a Publication of the IEEE Systems, Man, and Cybernetics Society, vol. 40, no. 3, pp. 939-950, 2010.

[8] Y.-J. Liu, J. Li, S. Tong, and C. L. P. Chen, "Neural network control-based adaptive learning design for nonlinear systems with full-state constraints," IEEE Transactions on Neural Networks and Learning Systems, vol. 27, no. 7, pp. 1562-1571, 2016.

[9] D.-P. Li, Y.-J. Liu, S. Tong, C. L. P. Chen, and D.-J. Li, "Neural networks-based adaptive control for nonlinear state constrained systems with input delay," IEEE Transactions on Cybernetics, vol. 49, no. 4, pp. 1249-1258, 2019.

[10] B. Chen, X. P. Liu, S. S. Ge, and C. Lin, "Adaptive fuzzy control of a class of nonlinear systems by fuzzy approximation approach," IEEE Transactions on Fuzzy Systems, vol. 20, no. 6, pp. 1012-1021, 2012.

[11] Y. Sun, B. Chen, C. Lin, H. Wang, and S. Zhou, "Adaptive neural control for a class of stochastic nonlinear systems by backstepping approach," Information Sciences, vol. 369, pp. 748-764, 2016.

[12] H. Wang, K. Liu, X. Liu, B. Chen, and C. Lin, "Neural-based adaptive output-feedback control for a class of nonstrictfeedback stochastic nonlinear systems," IEEE Transactions on Cybernetics, vol. 45, no. 9, pp. 1977-1987, 2015.

[13] H. Wang, X. Liu, K. Liu, and H. R. Karimi, “Approximationbased adaptive fuzzy tracking control for a class of nonstrictfeedback stochastic nonlinear time-Delay systems," IEEE Transactions on Fuzzy Systems, vol. 23, no. 5, pp. 1746-1760, 2015.

[14] S. Tong, Y. Li, and S. Sui, “Adaptive fuzzy output feedback control for switched nonstrict-feedback nonlinear systems with input nonlinearities," IEEE Transactions on Fuzzy Systems, vol. 24, no. 6, pp. 1426-1440, 2016.

[15] L. Wang, M. V. Basin, H. Li, and R. Lu, "Observer-based composite adaptive fuzzy control for nonstrict-feedback systems with actuator failures," IEEE Transactions on Fuzzy Systems, vol. 26, no. 4, pp. 2336-2347, 2018.

[16] J. Wu, B. Su, J. Li, X. Zhang, X. Li, and W. Chen, "Adaptive fuzzy control for full states constrained systems with nonstrict-feedback form and unknown nonlinear dead zone," Information Sciences, vol. 376, pp. 233-247, 2017.

[17] H. Li, L. Bai, Q. Zhou, R. Lu, and L. Wang, "Adaptive fuzzy control of stochastic nonstrict-feedback nonlinear systems with input saturation," IEEE Transactions on Systems, Man, and Cybernetics: Systems, vol. 47, no. 8, pp. 2185-2197, 2017.

[18] D. Q. Mayne, J. B. Rawlings, C. V. Rao, and P. O. M. Scokaert, "Constrained model predictive control: stability and optimality," Automatica, vol. 36, no. 6, pp. 789-814, 2000.
[19] Z. Sun, L. Dai, K. Liu, Y. Xia, and K. H. Johansson, "Robust MPC for tracking constrained unicycle robots with additive disturbances," Automatica, vol. 90, no. 90, pp. 172-184, 2018.

[20] E. Gilbert and I. Kolmanovsky, "Nonlinear tracking control in the presence of state and control constraints: a generalized reference governor," Automatica, vol. 38, no. 12, pp. 2063-2073, 2002.

[21] T. Zhang, M. Xia, and Y. Yi, "Adaptive neural dynamic surface control of strict-feedback nonlinear systems with full state constraints and unmodeled dynamics," Automatica, vol. 81, no. 81, pp. 232-239, 2017.

[22] T. Zhang, M. Xia, Y. Yi, and Q. Shen, "Adaptive neural dynamic surface control of pure-feedback nonlinear systems with full state constraints and dynamic uncertainties," IEEE Transactions on Systems, Man, and Cybernetics: Systems, vol. 47, no. 8, pp. 2378-2387, 2017.

[23] T. Zhang, M. Xia, and J. Zhu, "Adaptive backstepping neural control of state-delayed nonlinear systems with full-state constraints and unmodeled dynamics," International Journal of Adaptive Control and Signal Processing, vol. 31, no. 11, pp. 1704-1722, 2017.

[24] K. P. Tee, S. S. Ge, and E. H. Tay, "Barrier Lyapunov functions for the control of output-constrained nonlinear systems," Automatica, vol. 45, no. 4, pp. 918-927, 2009.

[25] K. P. Tee and S. S. Ge, "Control of nonlinear systems with partial state constraints using a barrier Lyapunov function," International Journal of Control, vol. 84, no. 12, pp. 20082023, 2011.

[26] Y.-J. Liu and S. Tong, "Barrier Lyapunov functions-based adaptive control for a class of nonlinear pure-feedback systems with full state constraints," Automatica, vol. 64, no. 64, pp. 70-75, 2016.

[27] K. Sachan and R. Padhi, "Barrier Lyapunov function based state-constrained control for a class of nonlinear systems," IFAC-PapersOnLine, vol. 51, no. 1, pp. 7-12, 2018.

[28] J. Huang, C. Wen, W. Wang, and Y.-D. Song, "Design of adaptive finite-time controllers for nonlinear uncertain systems based on given transient specifications," Automatica, vol. 69, no. 69, pp. 395-404, 2016.

[29] J. Wu, W. Chen, and J. Li, "Global finite-time adaptive stabilization for nonlinear systems with multiple unknown control directions," Automatica, vol. 69, no. 69, pp. 298-307, 2016.

[30] F. Wang, B. Chen, X. Liu, and C. Lin, "Finite-time adaptive fuzzy tracking control design for nonlinear systems," IEEE Transactions on Fuzzy Systems, vol. 26, no. 3, pp. 1207-1216, 2018.

[31] C. Wang and Y. Wu, "Finite-time tracking control for strictfeedback nonlinear systems with full state constraints," International Journal of Control, vol. 92, no. 6, pp. 1426-1433, 2017.

[32] S. Zhao, H. Liang, P. Du, and S. Qi, “Adaptive NN finite-time tracking control of output constrained nonlinear system with input saturation," Nonlinear Dynamics, vol. 92, no. 4, pp. 1845-1856, 2018.

[33] J. Xia, J. Zhang, W. Sun, B. Zhang, and Z. Wang, "Finite-time adaptive fuzzy control for nonlinear systems with full state constraints," IEEE Transactions on Systems, Man, and Cybernetics: Systems, vol. 49, no. 7, pp. 1541-1548, 2019.

[34] B. Cui, Y. Xia, K. Liu, and G. Shen, "Finite-time tracking control for a class of uncertain strict-feedback nonlinear systems with state constraints: a smooth control approach," IEEE Transactions on Neural Networks and Learning Systems, pp. 1-13, 2020. 
[35] J. Wu, W. Chen, F. Yang, J. Li, and Q. Zhu, "Global adaptive neural control for strict-feedback time-delay systems with predefined output accuracy," Information Sciences, vol. 301, pp. 27-43, 2014.

[36] W. Chen, S. S. Ge, J. Wu, and M. Gong, "Globally stable Adaptive backstepping neural network control for uncertain strict-feedback systems with tracking accuracy known," IEEE Transactions on Neural Network Learning Systems, vol. 26, no. 9, pp. 1842-1854, 2015.

[37] J. Wu, J. Li, and W. Chen, "Practical adaptive fuzzy tracking control for a class of perturbed nonlinear systems with backlash nonlinearity," Information Sciences, vol. 420, pp. 517-531, 2017.

[38] Y. Chang, Y. Wang, F. E. Alsaadi, and G. Zong, "Adaptive fuzzy output-feedback tracking control for switched stochastic pure-feedback nonlinear systems," International Journal of Adaptive Control and Signal Processing, vol. 33, no. 8, 2019.

[39] L. Ma, G. Zong, and X. Zhao, "Observed-based adaptive finitetime tracking control for a class of nonstrict-feedback nonlinear systems with input saturation," Journal of the Franklin Institute, vol. 357, no. 16, pp. 11518-11544, 2019.

[40] L. Ma, N. Xu, and X. Huo, "Adaptive finite-time outputfeedback control design for switched pure-feedback nonlinear systems with average dwell time," Nonlinear Analysis: Hybrid Systems, vol. 37, Article ID 100908, 2020.

[41] J. Cai, R. Yu, and B. Wang, "Decentralized event-triggered control for interconnected systems with unknown disturbances," Journal of the Franklin Institute, vol. 357, no. 3, pp. 1494-1515, 2019.

[42] D. Wang, Z. H. Peng, T. S. Li, X. Q. Li, and G. Sun, "Adaptive dynamic surface control for a class of uncertain nonlinear systems in pure-feedback form," in Proceedings of the. Joint 48th Conference on Decision and Control (CDC) Held Jointly with 2009 28th Chinese Control Conference, pp. 1956-1961, Shanghai, China, December 2009.

[43] T. P. Zhang, H. Wen, and Q. Zhu, "Adaptive fuzzy control of nonlinear systems in pure feedback form based on input-tostate stability," IEEE Transactions on Fuzzy Systems, vol. 18, no. 1, pp. 80-93, 2010.

[44] Z.-P. Jiang and L. Praly, "Design of robust adaptive controllers for nonlinear systems with dynamic uncertainties," Automatica, vol. 34, no. 7, pp. 825-840, 1998.

[45] T. Zhang, X. Shi, Q. Zhu, and Y. Yang, "Adaptive neural tracking control of pure-feedback nonlinear systems with unknown gain signs and unmodeled dynamics," Neurocomputing, vol. 121, pp. 290-297, 2013.

[46] X. N. Xia and T. P. Zhang, "Adaptive output feedback dynamic surface control of nonlinear systems with unmodeled dynamics and unknown high-frequency gain sign," Neurocomputing, vol. 143, pp. 312-321, 2014.

[47] Z. P. Jiang and D. J. Hill, "A robust adaptive backstepping scheme for nonlinear systems with unmodeled dynamics," IEEE Transactions on Automatic Control, vol. 44, no. 9, pp. 1705-1711, 1999.

[48] S. L. Niculescu, Delay Effects on Stabiblity: A Robust Control Approach, Springer-Verlay, London, UK, 2001.

[49] W. Michiels and S. L. Niculescu, Stability and Stabilization of Time-Delay Systems: an Eigenvalued-Based Approach, SIAM, Philadelphia, PA, USA, 2007.

[50] S. S. Ge, F. Hong, and T. H. Lee, "Adaptive neural network control of nonlinear systems with unknown time delays," IEEE Transactions on Automatic Control, vol. 48, no. 45, pp. 2004-2010, 2003.
[51] S. S. Ge, F. Hong, and T. H. Lee, "Adaptive neural control of nonlinear time-delay systems with unknown virtual control coefficients," IEEE Transactions on Systems, Man and Cybernetics, Part B (Cybernetics), vol. 34, no. 1, pp. 499-516, 2004.

[52] S. S. Ge and K. P. Tee, "Approximation-based control of nonlinear MIMO time-delay systems," Automatica, vol. 43, no. 1, pp. 31-43, 2007.

[53] F. Hong, S. S. Ge, and T. H. Lee, "Practical adaptive neural control of nonlinear systems with unknown time delays," IEEE Transactions on Systems, Man and Cybernetics, Part B (Cybernetics), vol. 35, no. 4, pp. 849-854, 2005.

[54] D. W. C. Ho, J. Li, and Y. Niu, "Adaptive neural control for a class of nonlinearly parametric time-delay systems," IEEE Transactions on Neural Networks, vol. 16, no. 3, pp. 625-635, 2005.

[55] S. Yu, X. Yu, B. Shirinzadeh, and Z. Man, "Continuous finitetime control for robotic manipulators with terminal sliding mode," Automatica, vol. 41, no. 11, pp. 1957-1964, 2005.

[56] Y. Wang, Y. Song, M. Krstic, and C. Wen, "Fault-tolerant finite time consensus for multiple uncertain nonlinear mechanical systems under single-way directed communication interactions and actuation failures," Automatica, vol. 63, no. 63, pp. 374-383, 2016.

[57] G. H. Hardy, J. E. Littlewood, and G. P“®lya, Inequalities, Cambridge University Press, Cambridge, UK, 1952.

[58] Y. Wang and Y. Song, "Fraction dynamic-surface-based neuroadaptive finite-time containment control of multiagent systems in nonaffine pure-feedback form," IEEE Transactions on Neural Networks and Learning Systems, vol. 28, no. 3, pp. 678-689, 2017.

[59] S. P. Bhat and D. S. Bernstein, "Finite-time stability of continuous autonomous systems," SIAM Journal on Control and Optimization, vol. 38, no. 3, pp. 751-766, 2000.

[60] B. Ren, S. S. Ge, K. P. Tee, and T. H. Lee, "Adaptive neural control for output feedback nonlinear systems using a barrier Lyapunov function," IEEE Transactions on Neural Network Learning Systems, vol. 21, no. 8, pp. 1339-1345, 2010. 